\title{
Aperture Excitation of Electrically Large, Lossy Cavities
}

David A. Hill John W. Adams Mark T. Ma Arthur R. Ondrejka Bill F. Riddle Myron L. Crawford Robert T. Johnk 



\title{
Aperture Excitation of Electrically Large, Lossy Cavities
}

David A. Hill

John W. Adams

Mark T. Ma

Arthur R. Ondrejka

Bill F. Riddle

Myron L. Crawford

Robert T. Johnk

\author{
Electromagnetic Fields Division \\ Electronics and Electrical Engineering Laboratory \\ National Institute of Standards and Technology \\ 325 Broadway \\ Boulder, Colorado 80303-3328
}

September 1993

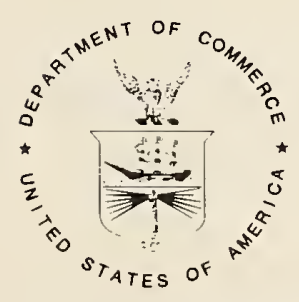

U.S. DEPARTMENT OF COMMERCE, Ronald H. Brown, Secretary TECHNOLOGY ADMINISTRATION, Mary L. Good, Under Secretary for Technology NATIONAL INSTITUTE OF STANDARDS AND TECHNOLOGY, Arati Prabhakar, Director 
National Institute of Standards and Technology Technical Note

Natl. Inst. Stand. Technol., Tech. Note 1361, 76 pages (September 1993) CODEN:NTNOEF

U.S. GOVERNMENT PRINTING OFFICE

WASHINGTON: 1993

For sale by the Superintendent of Documents, U.S. Government Printing Office, Washington, DC 20402-9325 
CONTENTS

Page

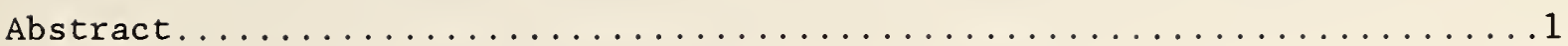

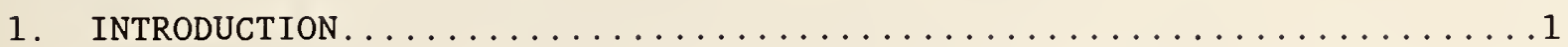

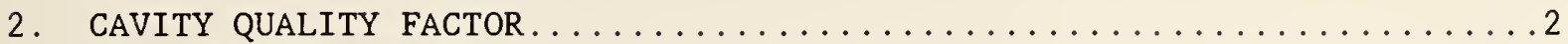

2.1 Wall Losses............................... . 4

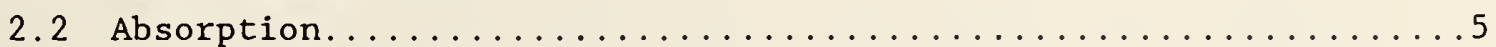

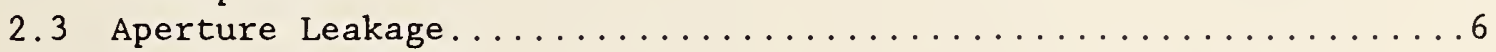

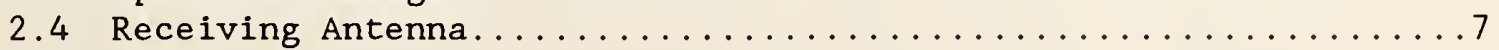

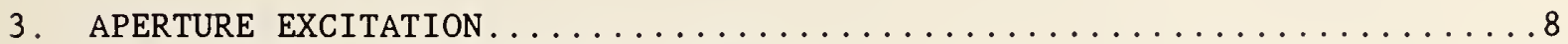

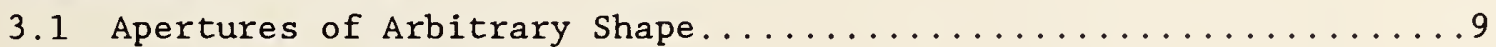

3.2 Circular Aperture.........................

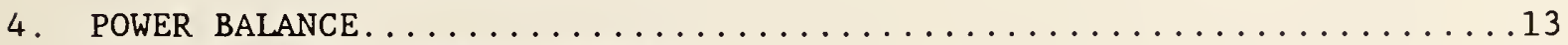

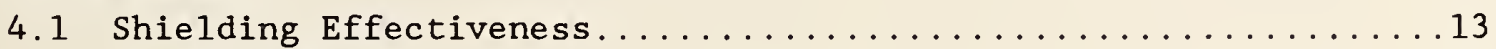

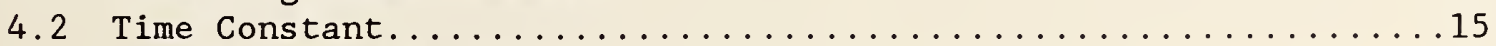

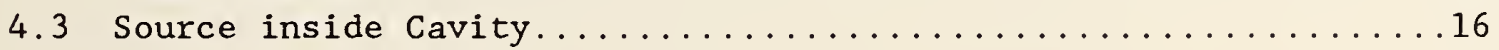

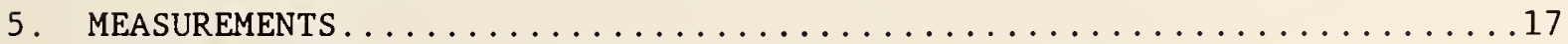

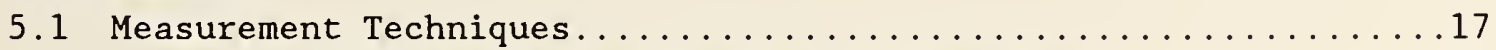

5.2 Comparison of Measured and Theoretical Results............21

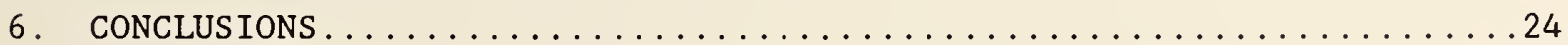

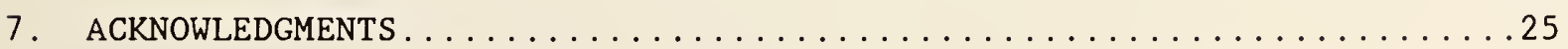

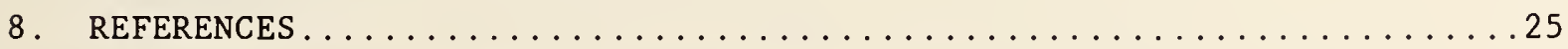

APPENDIX A. ABSORPTION BY A HOMOGENEOUS SPHERE..............27

APPENDIX B. TRANSMISSION CROSS SECTION OF A SMALL CIRCULAR APERTURE.... 34

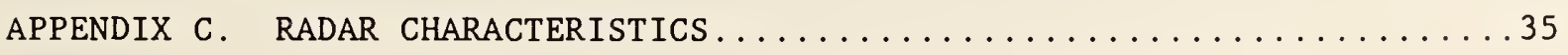

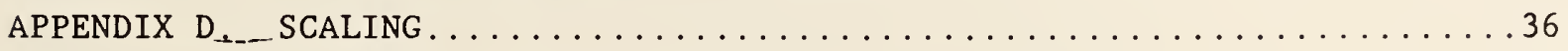

APPENDIX E. ELECTRICAL PROPERTIES OF SALT WATER.............. 41

APPENDIX $F$. SOFTWARE TO ANALYZE A RECTANGULAR CAVITY........... 41 



\title{
APERTURE EXCITATION OF ELECTRICALLY LARGE, LOSSY CAVITIES
}

David A. Hill, John W. Adams, Mark T. Ma, Arthur R. Ondrejka,

Bill F. Riddle, Myron L. Crawford, and Robert T. Johnk

\author{
Electromagnetic Fields Division \\ National Institute of Standards and Technology \\ Boulder, Colorado 80303
}

\begin{abstract}
We present a theory based on power balance for aperture excitation of electrically large, lossy cavities. The theory yields expressions for shielding effectiveness, cavity $Q$, and cavity time constant. In shielding effectiveness calculations, the incident field can be either a single plane wave or a uniformly random field to model reverberation chamber or random field illumination. The $Q$ theory includes wall loss, absorption by lossy objects within the cavity, aperture leakage, and power received by antennas within the cavity. Extensive measurements of shielding effectiveness, cavity $Q$, and cavity time constant were made on a rectangular cavity, and good agreement with theory was obtained for frequencies from 1 to $18 \mathrm{GHz}$.
\end{abstract}

Key words: absorption; aperture; cavity; polarizability; quality factor; reverberation chamber; scaling; shielding effectiveness; time constant; transmission cross section.

\section{INTRODUCTION}

In many electromagnetic interference (EMI) problems, the important electronic systems are located within a metal enclosure with apertures. In such cases it is important to know the shielding effectiveness (SE) of the enclosure so that we can relate the interior fields to the external incident fields. For example, the high intensity radiated fields (HIRFs) suggested in SAE draft advisory circular AE4R [1] are in the 1000 to $7000 \mathrm{~V} / \mathrm{m}$ range for frequencies above $400 \mathrm{MHz}$, the frequencies used by most radars. However, the shielding effectiveness of aircraft skins is not well known, so the interior fields that excite the aircraft electronics are not well characterized.

The purpose of this report is to develop a mathematical model for the shielding effectiveness of electrically large enclosures that contain 
apertures and interior loading. The method that we present uses a power balance approach and yields a simple, approximate expression for the average field strength throughout the cavity enclosed by the enclosure. This method does not yield the fine detail of the interior fields as would be obtained by a numerical method [2], but it has the advantages of being applicable to both $\mathrm{cw}$ and pulsed fields and not requiring all the geometrical details of the enclosure, the apertures, and the loads. The theory is similar to that developed for reverberation chambers [3-5], where the average power density is assumed to be uniform throughout the chamber.

The organization of this report is as follows. Section 2 contains a derivation for the cavity quality factor (Q) of an electrically large cavity where the losses include skin effect in the walls, volume absorption by lossy objects within the cavity, leakage through apertures, and power absorbed by receiving antennas. Section 3 covers aperture excitation of the cavity by an external field. Section 4 contains a power balance derivation of the shielding effectiveness and the time constant of the cavity. Section 5 presents measurement methods and comparisons of measured and theoretical values of $S E, Q$, and time constant. Section 6 presents conclusions and recommendations for further work. A number of appendices contain mathematical and software details.

\section{CAVITY QUALITY FACTOR}

The $Q$ of a cavity is a key quantity in calculating both SE of the surrounding shield and the time constant of the cavity. We consider an electrically large (multimode) cavity as shown in figure 1 . In general the cavity will have apertures and will contain lossy objects and at least one receiving antenna. The definition for $Q$ is

$$
Q=\omega U_{s} / P_{d},
$$

where $\omega$ is the excitation (angular) frequency, $U_{S}$ is the steady state energy in the cavity, and $\mathrm{P}_{\mathrm{d}}$ is the dissipated power. In our application the 
cavity is excited by an exterior field incident on apertures, but the $Q$ theory does not depend on the excitation method. Thus this theory is also applicable to reverberation chambers excited by antennas.

We can write the steady state energy $U_{S}$ as the product of the energy density $W$ times the cavity volume $\mathrm{V}$ :

$$
\mathrm{U}_{\mathrm{S}}=\mathrm{WV} \text {. }
$$

In eq (2) we assume that $W$ is uniform throughout the cavity volume, and this uniformity has been shown for the average energy density in reverberation chambers [4]. In reverberation chamber measurements, an ensemble average is obtained by rotating a mode stirrer, and in our theory we deal with ensemble averages that could represent averages over different cavity shapes. It is useful to write $W$ in terms of the RMS electric field E:

$$
W=\epsilon_{0} E^{2},
$$

where $\epsilon_{0}$ is the permittivity of free space and we have assumed the stored electric and magnetic energies are equal. $E^{2}$ should also be interpreted as an ensemble average. It is also useful to write an expression for the power density $S_{c}$ within the cavity:

$$
s_{c}=E^{2} / \eta_{0}=c W
$$

where $\eta_{0}=\left(\mu_{0} / \epsilon_{0}\right)^{1 / 2}, c=1 /\left(\mu_{0} \epsilon_{0}\right)^{1 / 2}$, and $\mu_{0}$ is the magnetic permeability of free space. $S_{c}$ should also be interpreted as an ensemble average.

The dissipated power can by written as the sum of four terms:

$$
P_{d}=P_{d 1}+P_{d 2}+P_{d 3}+P_{d 4}
$$


where $\mathrm{P}_{\mathrm{d} 1}$ is the power dissipated in the cavity walls, $\mathrm{P}_{\mathrm{d} 2}$ is the power absorbed in loading objects within the cavity, $\mathrm{P}_{\mathrm{d} 3}$ is the power lost through aperture leakage, and $\mathrm{P}_{\mathrm{d} 4}$ is the power dissipated in the loads of receiving antennas. The forms of eqs (1) and (5) suggest that we write an expression for the inverse of $Q$ :

$$
\mathrm{Q}^{-1}=\mathrm{Q}_{1}^{-1}+\mathrm{Q}_{2}^{-1}+\mathrm{Q}_{3}^{-1}+\mathrm{Q}_{4}^{-1}
$$

where $\mathrm{Q}_{1}=\omega \mathrm{U}_{\mathrm{s}} / \mathrm{P}_{\mathrm{d} 1}, \mathrm{Q}_{2}=\omega \mathrm{U}_{\mathrm{s}} / \mathrm{P}_{\mathrm{d} 2}, \mathrm{Q}_{3}=\omega \mathrm{U}_{\mathrm{s}} / \mathrm{P}_{\mathrm{d} 3}$, and $\mathrm{Q}_{4}=\omega \mathrm{U}_{\mathrm{s}} / \mathrm{P}_{\mathrm{d} 4}$.

The smallest of $Q_{1}, Q_{2}, Q_{3}$, and $Q_{4}$ will be the dominant contributor to $Q$. We now consider the individual loss mechanisms.

\section{1 Wall Losses}

For highly conducting walls the wall loss can be determined from the skin depth approximation. For reverberation chambers, the wall loss has been determined both by averaging over the individual cavity modes [6] and by averaging plane wave losses over all incidence angles and polarizations [5]. The latter approach is consistent with our ensemble average theory. In either case, the result for $Q_{1}$ is

$$
\mathrm{Q}_{1}=\frac{3 \mathrm{~V}}{2 \mu_{\mathrm{r}} \mathrm{S} \delta} \text {, where } \delta=\left(2 / \omega \mu_{\mathrm{w}} \sigma_{\mathrm{w}}\right)^{1 / 2}, \mu_{\mathrm{r}}=\mu_{\mathrm{w}} / \mu_{0} \text {, }
$$

where $S$ is the cavity surface area, $\delta$ is the skin depth, $\mu_{w}$ is the wall permeability, and $\sigma_{\mathrm{w}}$ is the wall conductivity.

Complications, such as variations in wall conductivity and permeability with position, could also be treated using the skin depth approximation, but the skin depth would then be a function of position. Wall coatings, such as 
paint, could also be included, but we would still lump all wall losses into $\mathrm{Q}_{1}$

\subsection{Absorption}

In general the absorption cross section $\sigma_{a}$ of a lossy object depends on the incidence angle and polarization of the incident plane wave [7]. Since we assume that the fields in an electrically large cavity can be written as a superposition of plane waves of all incidence angles and polarizations [8], the absorbed power can be written as the product of the cavity power density $\mathrm{S}_{c}$ and the averaged absorption cross section $\left\langle\sigma_{a}>\right.$ :

$$
\mathrm{P}_{\mathrm{d} 2}=\mathrm{S}_{\mathrm{c}}<\sigma_{\mathrm{a}}>
$$

where $\langle>$ indicates an incidence angle average over $4 \pi$ steradians and an average over all polarizations.

The absorption cross section in eq (9) can be that of a single object or a summation for a number of absorbers. For example, for $M$ absorbers $\left\langle\sigma_{a}\right\rangle$ in eq (9) would be replaced by:

$$
<\sigma_{a}>=\sum_{i=1}^{M}<\sigma_{a i}>
$$

where $\left\langle\sigma_{a i}\right\rangle$ is the averaged absorption cross section of the $i$ th absorber. For the example of an aircraft, the $i$ summation in eq (10) could represent absorption by people, seats, wiring, etc.

For the simple case of a uniform sphere, the absorption cross section is independent of the incidence angle and polarization of the incident field and no averaging is required. The cross section formulation for a uniform sphere is given in Appendix A. 
If we use eqs (2), (4), (7), and (9), we can derive the following expression for $\mathrm{Q}_{2}$ :

$$
\mathrm{Q}_{2}=\frac{2 \pi \mathrm{V}}{\lambda<\sigma_{\mathrm{a}}>}
$$

where $\lambda$ is the free-space wavelength. The frequency dependence of $Q_{2}$ can be fairly complicated because of the frequency dependence of $<\sigma_{a}>$. (See Appendix A for the frequency dependence of the absorption cross section of a homogeneous sphere.)

\subsection{Aperture Leakage}

In general the transmission cross section $\sigma_{\ell}$ of an aperture depends on the incidence angle and polarization of the incident plane wave [9]. We again assume that the fields can be written as a superposition of plane waves of all incidence angles and polarizations, but only the plane waves that propagate toward the apertures contribute to the transmitted power. Consequently, we introduce a factor of $1 / 2$ in the product expression for the leakage power:

$$
\mathrm{P}_{\mathrm{d} 3}=\mathrm{S}_{\mathrm{c}}<\sigma_{\ell}>/ 2
$$

where $<>$ again represents an average over incidence angle and polarization.

The transmission cross section in eq (12) can be that of a single aperture or a summation for a number of apertures. For $N$ apertures, $\left\langle\sigma_{\ell}\right\rangle$ in eq (12) would be replaced by

$$
<\sigma_{\ell}>=\sum_{i=1}^{N}<\sigma_{\ell i}>
$$


where $\left\langle\sigma_{\ell i}>\right.$ is the averaged transmission cross section of the $i$ th aperture. For the example of an aircraft, the $\mathrm{N}$ summation in eq (13) could represent leakage through windows and other apertures of different shapes and sizes. Specific expressions for transmission cross sections are given in section 3. If we use eqs (2), (4), (7), and (13), we can derive the following expression for $\mathrm{Q}_{3}$ :

$$
\mathrm{Q}_{3}=\frac{4 \pi \mathrm{V}}{\lambda<\sigma_{\ell^{\prime}}}
$$

For electrically large apertures, $\left\langle_{\ell}\right\rangle$ is independent of frequency, and $Q_{3}$ is proportional to frequency. For small or resonant apertures, the frequency dependence of $\mathrm{Q}_{3}$ is more complicated.

\subsection{Receiving Antenna}

The power $\mathrm{P}_{\mathrm{d} 4}$ dissipated in the load of the receiving antenna can be written as the product of the cavity power density and the averaged effective area of the receiving antenna:

$$
P_{d 4}=S_{c}<A_{e}>
$$

where the effective area $A_{e}$ is also averaged over incidence angle and polarization. The average effective area $\left\langle\mathrm{A}_{\mathrm{e}}\right\rangle$ of a lossless antenna can be written as the product of the effective area of an isotropic antenna $\left(\lambda^{2} / 4 \pi\right)$, the impedance mismatch factor $\mathrm{m}$, and a polarization mismatch factor of $1 / 2[10,11]$ :

$$
<A_{e}>=\frac{m \lambda^{2}}{8 \pi}
$$


$\left\langle A_{e}\right\rangle$ in eq (16) does not depend on the antenna pattern because it is averaged over all possible incidence angles and polarizations [8]. The impedance mismatch factor $m$ is equal to 1 for a matched load and is less than 1 otherwise.

If we use eqs (2), (4), (7), (15), and (16), we can derive the following expression for $\mathrm{Q}_{4}$ :

$$
Q_{4}=\frac{16 \pi^{2} V}{m \lambda^{3}}
$$

If there is more than one receiving antenna in the cavity, then $\left\langle A_{e}>\right.$ in eq (15) is replaced by a sum of averaged effective areas. For a matched load $(m=1), Q_{4}$ is proportional to frequency cubed. This means that $Q_{4}$ is small for low frequencies and is the dominant contributor to the total $Q$ in eq (6). The effect of antenna loading on the $Q$ of reverberation chambers has been observed experimentally [12]. At high frequencies, $Q_{4}$ becomes large and contributes little to the total Q.

\section{APERTURE EXCITATION}

Consider a cw plane wave of power density $\mathrm{s}_{i}$ incident on the shield apertures as shown in figure 1. If the total transmission cross section of the apertures is $\sigma_{t}$, the power $\mathrm{P}_{t}$ transmitted into the cavity is

$$
P_{t}=\sigma_{t} S_{i}
$$

(Of course power will also leak out through the apertures, but we lump that effect under leakage loss $\mathrm{P}_{\mathrm{d} 3}$ as discussed in Section 2.3.) For the general case of $N$ apertures, $\sigma_{t}$ can be written as a sum: 


$$
\sigma_{t}=\sum_{i=1}^{N} \sigma_{t i},
$$

where $\sigma_{t i}$ is the transmission cross section of the ith aperture. In general, $\sigma_{t i}$ and $\sigma_{t}$ depend on the frequency, incidence angle, and polarization of the incident field.

If the shielded cavity is illuminated in a reverberation chamber (random illumination), then the transmitted power can be written

$$
P_{t}=\left\langle\sigma_{t}>S_{i} / 2\right.
$$

The factor $1 / 2$ in eq (20) results from shadowing of the incident field by the electrically large enclosure and is a good approximation for convex shields. The average value of the transmission cross section for $N$ apertures is obtained directly from eq (19):

$$
\left.<\sigma_{t}\right\rangle=\sum_{i=1}^{N}<\sigma_{t i}>\text {. }
$$

The results in (20) and (21) could also be used in applications where the incidence angle and polarization are unknown.

\subsection{Apertures of Arbitrary Shape}

Consider a plane wave incident on an aperture in a perfectly conducting sheet as shown in figure 2. For convenience, we drop the subscript $i$ that identifies the ith aperture in the shield. Aperture theory has been developed primarily for apertures in flat, perfectly conducting screens of infinite extent and zero thickness [9]. Here we assume that the shield is locally planar and that the shield thickness is small. Aperture theory can be subdivided into three cases where the aperture dimensions are either small, comparable, or large compared to the wavelength. 
For electrically large apertures, the geometrical optics approximation yields

$$
\sigma_{t}=\mathrm{A} \cos \theta^{i}
$$

where $A$ is the aperture area and $\theta^{i}$ is the incident elevation angle. Thus $\sigma_{t}$ is independent of frequency, polarization, and azimuth of the incident field. For this case, the average transmission cross section can be written:

$$
<\sigma_{t}>=\frac{1}{2 \pi} \int_{0}^{2 \pi} \mathrm{d} \phi^{\mathrm{i}} \int_{0}^{\pi / 2} \mathrm{~d} \theta^{\mathrm{i}} \mathrm{A} \cos \theta^{\mathrm{i}} \sin \theta^{\mathrm{i}}=\mathrm{A} / 2
$$

where we restrict $\theta^{i}$ to angles less than $\pi / 2$ because the field is incident from only one side of the screen.

For electrically small apertures, polarizability theory states that the transmitted fields are those of induced electric and magnetic dipole moments $[9,13]$. This theory yields a transmission cross section that is proportional to frequency to the fourth power:

$$
\sigma_{t}=\mathrm{C} \mathrm{k}^{4}
$$

where $C$ depends on incident angle and polarization and aperture size and shape, but is independent of frequency. The wavenumber $k=\omega / c$. The specific form of $\mathrm{C}$ for a circular aperture will be given in the following section.

In the resonance region, the aperture dimensions are comparable to a wavelength, and the frequency dependence of $\sigma_{t}$ depends on the aperture shape. Numerical methods [9] can be used to compute $\sigma_{t}$ for such cases, but we will not pursue such methods here. 


\subsection{Circular Aperture}

The circular aperture is of particular interest because it has an analytical solution and it is easy to work with experimentally. The geometry for a circular aperture of radius a is shown in figure 3 . An exact solution for the transmission coefficient is available in terms of spheroidal functions [14], but we choose to construct a simpler solution in terms of the simple approximations that are available for electrically large and small circular apertures.

For electrically large circular apertures, the geometrical optics approximations in eqs (22) and (23) yield the following expressions for the transmission cross section and the averaged transmission cross section:

$$
\sigma_{t}=\pi a^{2} \cos \theta^{i} \text { and }\left\langle\sigma_{t}>=\pi a^{2} / 2\right.
$$

For electrically small circular apertures, polarizability theory [9] can be used to determine the effective electric and magnetic dipole moments and the resultant transmission cross section. The details are given in Appendix B. The transmission cross section depends on the polarization and the elevation angle of the incident field. For the electric field polarized parallel to the incidence plane (defined by the incident wave vector and the normal to the aperture), we write the transmission cross section as $\sigma_{\text {tpar }}$ :

$$
\sigma_{\text {tpar }}=\frac{64}{27 \pi} k^{4} a^{6}\left(1+\frac{1}{4} \sin ^{2} \theta^{i}\right)
$$

For perpendicular polarization, we write the transmission cross section as $\sigma_{\text {tperp }}:$

$$
\sigma_{\text {tperp }}=\frac{64}{27 \pi} \mathrm{k}^{4} \mathrm{a}^{6} \cos ^{2} \theta^{\mathrm{i}} .
$$


Both $\sigma_{\text {tpar }}$ and $\sigma_{\text {tperp }}$ have the $\mathrm{k}^{4}$ dependence given by eq (24), and they are equal for normal incidence $\left(\theta^{i}=0\right)$. We assume that an incident random field will have equal power densities in the parallel and perpendicular waves. Thus the averaged transmission cross section can be written

$$
<\sigma_{t}>=\frac{1}{2} \int_{0}^{\pi / 2} \mathrm{~d} \theta^{\mathrm{i}}\left(\sigma_{\text {tpar }}+\sigma_{\text {tperp }}\right) \sin \theta^{\mathrm{i}}
$$

where we have used the fact that the transmission cross sections are independent of the incident azimuth angle. If we substitute eqs (26) and (27) into eq (28) and carry out the integration over $\theta^{i}$, we obtain

$$
<\sigma_{t}>=\frac{16}{9 \pi} \mathrm{k}^{4} \mathrm{a}^{6}
$$

We do not have a simple expression for the transmission cross section that is valid in the resonance region, but the circular aperture does not have strong resonances [15]. Thus we choose to cover the entire frequency range by using only the electrically small and electrically large approximations. The crossover wavenumber $k_{c}$ where we switch from eq (29) to eq (25) for the average transmission cross sections is given by equating eqs (25) and (29):

$$
\pi a^{2} / 2=\frac{16}{9 \pi} k_{c}^{4} a^{6}
$$

The solution to eq $(30)$ is

$$
\mathrm{k}_{\mathrm{c}} \mathrm{a}=\left(9 \pi^{2} / 32\right)^{1 / 4} \approx 1.29
$$

This technique is not valid for long, narrow apertures, which typically have strong resonances. 


\section{POWER BALANCE}

\subsection{Shielding Effectiveness}

Consider again the geometry in figure 1, where an incident wave is incident on a shielded cavity with apertures. We wish to determine the power density $S_{c}$ inside the cavity. For steady state conditions, we require that the power $P_{t}$ transmitted through the apertures is equal to the power $\mathrm{P}_{\mathrm{d}}$ dissipated in the four loss mechanisms considered in Section 2:

$$
P_{t}=P_{d}
$$

If we substitute eqs (1), (2), (4), and (18) into (32), we can solve for the power density $s_{c}$ in the cavity:

$$
s_{c}=\frac{\sigma_{t} \lambda Q}{2 \pi V} s_{i}
$$

Since we have assumed that the power density $s_{c}$ is uniform throughout the cavity, we can define shielding effectiveness in terms of the ratio of the incident and cavity power densities:

$$
\mathrm{SE}=10 \log _{10}\left(\mathrm{~S}_{\mathrm{i}} / \mathrm{S}_{\mathrm{c}}\right)=10 \log _{10}\left(\frac{2 \pi \mathrm{V}}{\sigma_{t} \lambda \mathrm{Q}}\right), \mathrm{dB}
$$

The results in eqs (33) and (34) are consistent with a recent treatment of a related problem [16]. We have defined SE to be a positive number when the cavity power density is less than the incident power density. Our definition of $\mathrm{SE}$ in (34) depends on the cavity volume and $Q$ in addition to the transmission cross section $\sigma_{t}$. For the application of measuring $S E$ of 
materials covering the aperture, it would be more logical to work directly with $\sigma_{t}$.

The results in eqs (33) and (34) apply to a single incident plane wave where $\sigma_{t}$ depends on the incident direction and polarization. For the case of uniformly random incidence (as in a reverberation chamber), we need to replace $\sigma_{t}$ in eqs (33) and (34) by one-half the averaged value $\left\langle\sigma_{t}\right\rangle / 2$.

The $Q$ enhancement of the cavity power density is clear in eqs (33) and (34), and we can see that a lossy cavity (low Q) has a greater shielding effectiveness than a high $Q$ cavity. The importance of loss is seen if we consider the special case where the cavity is $\operatorname{los} \operatorname{less}\left(\mathrm{P}_{\mathrm{d} 1}=\mathrm{P}_{\mathrm{d} 2}=\mathrm{P}_{\mathrm{d} 4}=0\right)$ except for leakage. In this case $Q$ is given by

$$
\mathrm{Q}=\mathrm{Q}_{3}=\frac{4 \pi \mathrm{V}}{\lambda<\sigma_{\ell}>}
$$

If we substitute eq (35) into eq (33), we obtain

$$
s_{c}=s_{i} \frac{2 \sigma_{t}}{<\sigma_{\ell}>} .
$$

For the case of uniformly random excitation, the transmission cross zection is replaced by one half the averaged cross section. However, the averaged transmission cross section is equal to the averaged leakage leakage cross section $\left(\left\langle\sigma_{t}\right\rangle=\left\langle\sigma_{\ell}\right\rangle\right)$, and eq (36) reduces to

$$
\mathrm{S}_{\mathrm{c}}=\mathrm{S}_{\mathrm{i}} \text { or } \mathrm{SE}=0 \mathrm{~dB} \text {. }
$$

Thus the leakage loss equals the transmitted power, and the cavity has zero shielding. We expect aircraft cavities to have significant loss and fairly low Q. 


\subsection{Time Constant}

Up to this point we have considered only steady-state, single-frequency excitation. Since radar pulses are important in aircraft applications, we also need to consider transient effects. In general this is a complex problem that is best handled using Fourier integral techniques. However, we can analyze the special case of a turned-on or turned-off sinusoid in a simpler manner.

We consider first the case of field decay where the source (the incident power density) is instantaneously turned off. By equating the change in the cavity energy $U$ to the negative of the dissipated power times a time increment $d t$, we obtain the differential equation

$$
\mathrm{dU}=-\mathrm{P}_{\mathrm{d}} \mathrm{dt}
$$

We can use eq (1) to replace $P_{d}$ in eq (38):

$$
d U=-(\omega U / Q) d t=-\frac{U}{\tau} d t
$$

where the time constant $\tau=Q / \omega$. The initial condition is $U=U_{S}$ at $t=0$. The solution of eq (39) with this initial condition is

$$
U=U_{s} e^{-t / \tau}, t>0
$$

The closely related case of a turned-on (step modulated) incident power density involves the same exponential function and time constant:

$$
U=U_{s}\left(1-e^{-t / \tau}\right), t>0
$$

The cavity energy density and power density also follow the same exponential variation with the same time constant, and eqs (40) and (41) agree with [3]. If a radar pulse duration is long compared to $\tau$, then the cavity fields will 
reach their steady-state value. However, if the pulse length is short compared to $\tau$, the fields will not reach their steady-state values before the incident pulse is turned off. Some common radars and their pulse characteristics are described in Appendix $C$.

A high $Q$ (long $\tau$ ) cavity might have poor steady-state SE, but would require a long time for the cavity fields to reach their steady state values. Physically, a high $Q$ (long $\tau$ ) means that waves make many bounces within the cavity before they decay.

\subsection{Source inside Cavity}

We can use the power balance theory of Section 4.1 to analyze the case of an internal source, a transmitting antenna located within the cavity. This configuration is of interest in calculating either the power density within an aircraft caused by unintentional emitters or the power density within a reverberation chamber with loading.

If a cw source within the cavity transmits power $P_{t}$, then eq (32) still holds, but $P_{t}$ now represents antenna transmitted power rather than aperture transmitted power. Using eqs (1), (2), (4), and (32), we can solve for the power density $S_{c}$ within the cavity:

$$
\mathrm{s}_{\mathrm{c}}=\frac{\lambda \mathrm{QP}}{2 \pi \mathrm{V}}
$$

Equation (42) applies to cavities with general loading because Q can include any of the loss mechanisms discussed in Section 2.

If an impedance-matched, receiving antenna is placed within the cavity, then the received power $P_{r}$ is the product of the effective area $\lambda^{2} / 8 \pi$ and the power density $S_{C}$ as discussed in Section 2.4. Using eq (42), we obtain the following expression for the received power: 


$$
P_{r}=\frac{\lambda^{3} Q}{16 \pi^{2} V} P_{t}
$$

If we solve eq (43) for $Q$, then we see that the cavity $Q$ can be determined from a measurement of the power ratio $\mathrm{P}_{\mathrm{r}} / \mathrm{P}_{t}$ :

$$
Q=\frac{16 \pi^{2} V}{\lambda^{3}} \frac{P_{r}}{P_{t}} .
$$

Equation (44) is commonly used for measuring the $Q$ of reverberation chambers [17] .

5. MEASUREMENTS

\subsection{Measurement Techniques}

A relatively small, rectangular cavity (which we call the FAA cavity) was constructed and used for measurements to validate the theoretical model. The theoretical model can be used for any size or shape of electrically large cavity. The physical cavity was selected to satisfy a number of practical limitations and yet to allow evaluation of all loss mechanisms in the theoretical model. Scaling considerations for general, lossy materials are discussed in Appendix D. The cavity was rectangular, $0.514 \mathrm{~m} \times 0.629 \mathrm{~m}$ $\times 1.75 \mathrm{~m}$. The cavity was selected to be large enough to provide sufficient mode density above $1 \mathrm{GHz}$, but to be light enough to meet weight requirements of our automated positioner. The three parameters that needed to be measured were quality factor $Q$, time constant $\tau$, and shielding effectiveness $\mathrm{SE}$, from 1 to $18 \mathrm{GHz}$ for various loss and/or loading conditions due to walls, absorption, antenna, and aperture.

of the various materials considered (galvanized steel, copper, and aluminum), aluminum was selected because it has a high electrical conductivity and is easy to weld. A removable panel was considered as an 
access door, but was rejected because of the variability of contact resistance and possible unknown loss in rf gasketing material. Because of the difficulty in welding and grinding open an end wall each time access was needed to the cavity, a minimum of opening was planned.

Double-ridged waveguide horns were selected because of their wide frequency range, 1 to $18 \mathrm{GHz}$. A stirrer and drive motor were installed inside the chamber to provide mode stirring. The stirrer was also made of aluminum. The drive motor was enclosed inside a high-isolation aluminum case so that motor noise would not escape into the cavity.

$Q$ was measured before any aperture was cut in a wall just to ensure that antennas, cables, and connectors were functioning properly and also to ensure the integrity of the welded seams. A second set of measurements was made with an aperture of radius $0.014 \mathrm{~m}$ cut in a side wall. This allowed coupling energy into and out of the cavity. Later a sphere of radius 0.066 $m$ and containing salt water was put inside the cavity. Still later, a set of three spheres of radius $0.066 \mathrm{~m}$ and containing salt water was put into the cavity. The salt water was selected as having absorption similar to a human body. The salt concentration was selected as that of ocean water so that we could use the electrical properties of salt water given by Saxton and Lane [18]. These electrical properties are discussed in Appendix E. Finally, everything was removed and two standard-gain horns were used in place of the double-ridged waveguide horn antennas. The aperture, of course, remained. This last step had to be added to try to quantify the loss of the double-ridged waveguide horn antennas, since there was greater loss measured than calculated. The standard gain horns have an efficiency of about 0.98 .

Initial measurements of Q relied on eq (44), previously given by Corona et a1. [17]. Volume and frequency errors introduce very little uncertainty, but the power ratio $\mathrm{P}_{\mathrm{r}} / \mathrm{P}_{t}$ requires consistent use of either maximum or average values. The power transmitted is determined at the same time cable loss is measured, by shorting the cables to the two antennas together. This removes all stirring effects and maximum and average values are within 0.1 $\mathrm{dB}$, as expected for $\mathrm{cw}$ excitation. The received power is coupled through the resonant cavity modes, and the stirrer introduced a 40 to $50 \mathrm{~dB}$ excursion, including 6 to $10 \mathrm{~dB}$ differences between maximum and average. 
The maximum-average difference must be known if maximum values are to be used. The most practical way to avoid this is to use average values. Perhaps later, when the distributions have been adequately characterized, this requirement can be relaxed.

For measurement of shielding effectiveness where there are two chambers, two stirrers, and two similar antennas, either maximum or average values might be used since both receiver powers are coupled through the resonant, stirred environment. However, there is a difference in type of distribution and maximum-average ratio of the two chambers, and averagebased SE values are a few decibels higher than maximum-based values. The observed maximum-average ratio is also higher in the outer-to-inner chamber distribution than in the larger, outer chamber distribution. Therefore, it is probably safer to use average values.

The measure of shielding effectiveness of the cavity was determined by measurements using the setup in figure 4. This was a direct measurement of two powers. The procedure for measuring the loss within the FAA cavity is similar, except the cable to the transmit antenna is connected to the transmit antenna in the FAA cavity. This is a modification to figure 4. The stirrer must be turning and the frequency sweep must be slow enough to allow one revolution of the stirrer at each frequency. The average power loss is the preferred parameter to measure. Cable losses were also measured and corrections were applied. A power-series, least-squares fit was obtained from the loss-frequency curve to use in eq (44) to obtain a smooth curve for $Q$ from loss measurements.

The measurement of the time constant required the setup shown in figure 5. This is described previously by Crawford et al. [19]. A burst of rf energy was provided for a microsecond and the maximum and average decay responses were recorded for each of 200 stirrer positions. The average decay for these 200 tuner positions was used to provide a clean decay response as shown in figure 6. An exponential least-squares fit was applied to determine the actual time constant. This provides a second measure of $Q$, since $Q$ is the product of angular frequency and time constant as indicated in Section 4.2.

Many factors that affect the measured values; not all are included in the theoretical model. The method of recording and processing data had to 
be varied from the procedure used for routine electromagnetic susceptibility measurements.

The usual procedure for measuring loss, fields, or shielding effectiveness in a mode-stirred chamber is to use a 'max hold' feature of the spectrum analyzer and record the maximum field strength during one or more frequency sweeps. The theoretical model in this work uses average values, so questions concerning the type of distribution and the relation between maxima and averages are relevant. Previous measurements [19] for relatively high-Q, mode-stirred chambers indicated a nominally $8 \mathrm{~dB}$ difference between maximum and average values of a distribution at any single frequency. The range of this difference was between 6.5 and $9.5 \mathrm{~dB}$.

Despite careful measurements by all of the several methods used, we measured substantially lower values of $Q$ than were predicted by our model. This indicated measurement errors, substantially higher losses than predicted, or model error. Investigations revealed a combination of the first two.

The measurement error was caused mainly by loss in the antennas. Double-ridged waveguide horn antennas were used initially since they have a large frequency range, $1-18 \mathrm{GHz}$. Subsequent measurements using standardgain horns with very high efficiency over a more-limited frequency range indicated about $2 \mathrm{~dB}$ less loss per antenna than had been measured using the double-ridged waveguide horns. This loss may not be the same in other applications where surface areas of the horn and mounting structures and the plastic parts are not so strongly excited.

Other additional losses could not be quantified as clearly in all cases, but each loss was demonstrated. By making reasonable estimates, we were able to show agreement between measured and calculated values within $20 \%$, which indicates validity of the model.

One of the unaccounted losses was due to lower-than-expected conductivity of aluminum. We initially used a value near that of pure aluminum from a reference handbook, but discovered that the metal actually used was an alloy with about half the conductivity of pure aluminum. An additional decrease in conductivity was measured at NIST using a parallelplate dielectric resonator technique [20]. This measured value $\left(8.83 \times 10^{6}\right.$ $\mathrm{S} / \mathrm{m}$ ) indicated an additional factor-of-2 decrease in conductivity. Whether 
this is due to the oxide layer, surface roughness, or other factors has not been determined. This total factor of 4 reduces the predicted Q by $4^{1 / 2}$ or 2 (see eq (8)).

Another substantial unaccounted loss was the wall loss due to additional surface area of cables, mounting brackets, antennas, stirrer, stirrer motor case, and bulkhead connectors. This additional surface area can be approximated, but the loss is difficult to model since the conductivities and current distributions are not known. We estimated 10 to 20 \& additional surface area depending on the complexity and size of the loading and antennas used. One more unaccounted factor that changes the calculated $Q$ is the reduction of the volume within the cavity. This was estimated to be 5 to $10 \%$. Again this depends on the size and complexity of everything within the cavity.

There is one other minor loss due to absorption of the dielectric cover on the cables within the chamber. We originally used semi-rigid (solid metal) coaxial transmission lines, but the mating, precision connectors are very susceptible to loosening due to vibration. Only a slight loosening causes a large mismatch. We changed to flexible, dielectric-covered cables and special connectors that were more reliable in this application.

During the absorptive-loading modeling, there were additional losses due to flexible tubing, corks, glassware, and support structures of foam. None of these corrections is dominant; different loading cases require different corrections. All of these loss mechanisms will be even more prevalent in most applications, especially in aircraft compartments.

\subsection{Comparison of Measured and Theoretical Results}

Samples of measured and theoretical results with four loading conditions are shown. The theoretical results were obtained from the computer program described in Appendix F. Previous estimates of field uncertainties in reverberation chambers excited by sources within the chamber $[4,19]$ range from \pm 4 to $\pm 8 \mathrm{~dB}$. Consequently, we consider agreement between measurements and theory within $\pm 4 \mathrm{~dB}$ to be good and within $\pm 8 \mathrm{~dB}$ to be acceptable. 
The results for the (relatively) empty cavity with an aperture of diameter $0.014 \mathrm{~m}$, but with no corrections applied, are shown in figures 7-9. Figure 7 shows the several calculated Q values based on the appropriate loss mechanism, the composite $Q$, and the measured $Q s$ by both loss and time constant. Figure 8 shows calculated and measured time constant, while figure 9 shows measured and calculated SE. The $Q$ values in figure 7 measured by the transmission loss method, eq (44), and the time constant method, eq (39), are in good agreement, but are much smaller than the theoretical values. This is typical of earlier reverberation chamber measurements [4,19]. The differences between measured and calculated values of $\tau$ and SE are also substantial. At first, the reasons for these differences were not known.

Next, the cavity with the $0.014 \mathrm{~m}$ aperture was loaded with one of the spheres containing salt water. In these and subsequent figures, conductivity, volume, and surface corrections have been applied. Figure 10 shows Qs due to three of the four loss mechanisms plus the composite $Q$ and the two measured Qs; the curves along the bottom are too compressed to read. $\mathrm{Q}_{1}$, due to wall loss, has dropped from that in figure 7 because we are using the measured wall conductivity $\left(8.83 \times 10^{6} \mathrm{~s} / \mathrm{m}\right) . \mathrm{Q}_{3}$, due to aperture leakage, reaches a minumim at the crossover from low to high frequency behavior as given by eq (31). Figure 11, an expanded view of figure 10, shows the part of the figure that has the Qs due to the dominant loss mechanisms plus the composite $Q$ and the two measured Qs. Figure 12 shows measured and calculated time constants plots. Figure 13 shows measured and calculated SE plots. There is much better agreement between theoretically predicted and measured values, but the disagreement indicated the need for further investigation.

Next we loaded the cavity with three spheres containing salt water. The results are shown in figures 14-17. Figure 14 shows three of the $Q$ curves; figure 15 shows the expanded portion that has the Qs with the dominant loss mechanisms, the composite $Q$, and the measured Qs. Figure 16 shows the time constant plots, and figure 17 shows the SE plots. Again, the agreement is good, but not good enough. 
The last set of figures, 18-21, covers a limited frequency range (12-18 $\mathrm{GHz}$ ), but reveals the missing loss factor. For these we used a set of standard-gain, $\mathrm{K}_{\mathrm{u}}$-band horns. The antennas have a fairly high efficiency, about 0.98 . These results show quite acceptable agreement between predicted and measured results. This indicates a poorer efficiency for the doubleridge waveguide horns than we had expected. Figure 18 shows the Qs; figure 19 shows the expanded Q curves. The agreement between theoretical and measured $Q$ is much better than that typically obtained for reverberation chambers [4]. Figure 20 shows the time constants, and figure 21 shows the SE values.

Shielding effectiveness measurements were also performed by Hatfield [21] on a rectangular cavity with a circular aperture in the Naval Surface Warfare Center (NSWC) reverberation chamber. The cavity contains a broadband, double-ridged receiving antenna and a paddle wheel (mode stirrer) as shown in figure 4. The box is made of the same aluminum alloy $\left(\sigma_{\mathrm{w}}=8.83 \times 10^{6} \mathrm{~s} / \mathrm{m}\right.$ and $\left.\mu_{\mathrm{r}}=1\right)$ with dimensions: $\ell=1.213 \mathrm{~m}, \mathrm{w}=0.603 \mathrm{~m}$, and $\mathrm{h}=0.937 \mathrm{~m}$. Two different aperture radii, $\mathrm{a}=2.94 \mathrm{~cm}$ and $3.51 \mathrm{~cm}$, were used.

Shielding effectiveness' measurements were made from $200 \mathrm{MHz}$ to $18 \mathrm{GHz}$ for both apertures. A comparison with calculated SE values is shown in figure 23. The theory is not expected to be valid below $400 \mathrm{MHz}$ because the box is not electrically large (the mode density is too low). The measured values show rapid frequency variations which do not appear in the smooth theory, but the rapid frequency variations are typical of reverberation chamber measurements [4]. The general agreement between theory and measurements is good above $400 \mathrm{MHz}$ for both aperture sizes. The smaller aperture yields greater SE, but the high frequency theoretical SE is low for both apertures. The reason for the decrease in SE with frequency is the increase in both cavity $Q$ and transmission cross section with frequency. Equation (34) shows the dependence of SE on these two quantities.

The measured results presented in this section could be scaled up in size and down in frequency to more nearly match the dimensions of aircrafts. However, the electrical properties of the walls and absorbers would also 
need to be scaled; the required scaling relationships are discussed in Appendix D.

\section{CONCLUSIONS}

We have presented a theory based on power balance for aperture excitation of electrically large, lossy cavities. The theory yields expressions for shielding effectiveness, cavity $Q$, and cavity time constant, but does not give the fine structure of the fields (standing waves, etc.) within the cavity. The advantage of the theory is that it does not require the detailed geometry of the cavity, absorbers, and apertures, but only requires a few defining parameters (cavity volume and surface area, wall conductivity, aperture transmission cross section, and absorption cross section of objects within the cavity).

The theory actually yields ensemble averages for quantities of interest ( $\mathrm{SE}, \mathrm{Q}$, and $\tau$ ), and the ensemble can be considered to represent different cavity geometries. Experimentally the ensemble average is obtained by use of a mode stirrer within the cavity, and that is how the experimental data for SE, Q, and $\tau$ were obtained in Section 5. The agreement of the theory with the broadband experimental data was good, but a great deal of effort was required to account for additional losses that lowered measured $Q$ and $\tau$ and increased measured SE. The theory and measurements of $Q$ and $\tau$ have application to improved characterization of reverberation chambers $[4,19]$ where the losses have been difficult to calculate.

A number of other extensions that would be useful. Measurements for cavities with multiple apertures would provide additional checks for the theory and would more nearly model typical aircraft geometries. For modeling aircraft windows, the effects of window glass (and possibly coatings) should be included in future calculations. A fifth loss mechanism, water vapor absorption, could be added to the $Q$ theory. This loss has been observed in reverberation chamber measurements above $18 \mathrm{GHz}$ [19]. Measurements with plane wave excitation in an anechoic chamber would provide additional checks for the angular and polarization features of the 
aperture theory. Also, measurements on an actual aircraft would be useful in determining whether additional features need to be added to the model.

Further experimental and theoretical studies of the statistical properties of fields in large cavities need to be performed. Such studies would be useful in extending the present theory to include probability density and distribution functions [22] in addition to average values. Some theoretical work on the statistical properties of fields in cavities [23,24] has been done, but a more complete statistical electromagnetic theory is needed.

\section{ACKNOWLEDGMENTS}

This research was supported by the Federal Aviation Administration. We would like to thank Mike Hatfield, NSWC, for providing shielding effectiveness data for the NSWC cavity and Richard Geyer, NIST, for performing the wall conductivity measurement for the FAA cavity.

\section{REFERENCES}

[1] Guidance to certification of Aircraft electrical/electronic systems for operation in the high intensity radiated fields (HIRF) environment, Society of Automotive Engineers, SAE AE4R Committee Report (July, 1991).

[2] Taflove, A.; Umashankar, K. A hybrid moment method/finite-difference time-domain approach to electromagnetic coupling and aperture penetration into complex geometries. IEEE Trans. Ant. Propagat., AP-30: $617-627,1982$.

[3] Richardson, R.E. Mode-stirred chamber calibration factor, relaxation time, and scaling laws. IEEE Trans. Instrum. Meas., IM-34: 573-580; 1985 .

[4] Crawford, M.L.; Koepke, G.H. Design, evaluation, and use of a reveberation chamber for performing electromagnetic susceptibility/vulnerability measurements. Nat. Bur. Stand. (U.S.) Tech. Note 1092; 1986.

[5] Dunn, J.M. Local, high-frequency analysis of the fields in a modestirred chamber. IEEE Trans. Electromag. Compat., EMC-32: 53-58; 1990. 
[6] Liu, B.H.; Chang, D.C.; Ma, M.T. Eigenmodes and the composite quality factor of a reverberating chamber. Nat. Bur. Stand. (U.S.) Tech. Note $1066 ; 1983$.

[7] Van de Hulst, H.C. Light Scattering by Small Particles. New York: Dover, 1981.

[8] Hill, D.A.; Crawford, M.L.; Kanda, M.; Wu, D.I. Aperture coupling to a coaxial air line: theory and experiment. IEEE Trans. Electromag. Compat.: $69-74 ; 1993$.

[9] Butler, C.M.; Rahmat-Samii, Y.; Mittra, R. Electromagnetic penetration through apertures in conducting surfaces. IEEE Trans. Ant. Propagat., AP-26: 82-93; 1978.

[10] Tai, C.T. On the definition of effective aperture of antennas. IEEE Trans. Ant. Propagat., AP-9: 224-225; 1961.

[11] Hill, D.A.; Francis, M.H. Out-of-band response of antenna arrays. IEEE Trans. Electromag. Compat., EMC-29: 282-288; 1987.

[12] Loughry, T.A. Frequency stirring: an alternate approach to mechanical mode-stirring for the conduct of electromagnetic susceptibility testing. Phillips Laboratory, Kirtland Air Force Base, NM, PL-TR--91$1036 ; 1991$.

[13] Bethe, H.A. Theory of diffraction by small holes. Phys. Rev., 66: 163$182 ; 1944$.

[14] Meixner, J.; Andrejewski, W. Strenge theorie der beugung ebener elektromagnetischer wellen an der vollkommen leitenden kreisscheibe und an der kreisformigen offnung im vollkemmen leitenden eben schirm, Annalen der Physik, 7: 157-168; 1950.

[15] Levine, H.; Schwinger, J. On the theory of electromagnetic wave diffraction by an aperture in an infinite plane conducting screen. Comm. Pure App1. Math., 3: 355-391; 1950.

[16] Lee, K.S.H.; Yang, F.-C.; Trends and bounds in RF coupling to a wire inside a slotted cavity. IEEE Trans. Electromag. Compat., EMC-34: 154$160 ; 1992$.

[17] Corona, P.; Latmiral, G.; Paolini, E. Performance and analysis of a reverberating enclosure with variable geometry. IEEE Trans. Electromag. Compat., EMC-22: 2-5; 1980.

[18] Saxton, J.A.; Lane, J.A. Electrical properties of sea water. Wireless Engineer, 29: 269-275; 1952.

[19] Crawford, M.L.; Ma, M.T.; Ladbury, J.M.; Riddle, B.F. Measurement and evaluation of a TEM/reverberating chamber. Nat. Inst. Stand. Tech. (U.S.) Tech. Note $1342 ; 1990$. 
[20] Geyer, R.G., National Institute of Standards and Technology, Boulder, CO. Private communication.

[21] Hatfield, M., Naval Surface Warfare Center, Dahlgren, VA. Private communication.

[22] Kostas, J.G.; Boverie, B. Statistical model for a mode-stirred chamber. IEEE Trans. Electromag. Compat., EMC-33: 366-370; 1991.

[23] Price, R.H.; Davis, H.T.; Bonn, R.H.; Wenaas, E.P.; Achenbach, R.; Gieri, V.; Thomas, R. ; Alcala, J. ; Hanson, J. ; Haynes, W. ; McCrea, C. ; Montano, C.; Peterson, R.; Trautlein, B.; Umber, R. Determination of the statistical distribution of electromagnetic field amplitudes in complex cavities, JAYCOR Report 88JAL129; 1988.

[24] Lehman, T.H. A statistical theory of electromagnetic fields in complex cavities. EMP Interaction Note 494; May 1993.

[25] Mie, G. Ann. Physik, 25: 377; 1908.

[26] Harrington, R.F. Time-Harmonic Electromagnetic Fields. New York: McGraw-Hill; 1961.

[27] Rumsey, V.H. Frequency Independent Antennas. New York: Academic Press; 1966.

[28] Hill, D.A. Electromagnetic scattering by buried objects of low contrast. IEEE Trans. Geosci. Rem. Sens., GRS-6: 195-203; 1988.

[29] Hasted, J.B. Aqueous Dielectrics. London: Chapman and Ha11; 1973, Ch. 6 , Electrolytic solutions.

APPENDIX A. ABSORPTION BY A HOMOGENEOUS SPHERE

The absorption loss due to loading objects inside a cavity can be written as the product of cavity power density and absorption cross section of the object. To simplify the analysis and facilitate experimental verifications, we assume that a loading object takes the form of a homogeneous sphere made of sea water to simulate a human.

An incident field with known frequency and intensity propagates toward the sphere. The problem at hand is then to determine the field penetrating into the sphere. The classical solution of this problem is due to Mie [25], based on the formulation of a vector wave equation,

$$
\nabla^{2} \overline{\mathrm{E}}+\mathrm{k}^{2} \mathrm{~m}^{2} \overline{\mathrm{E}}=0
$$


in a source-free region satisfying appropriate boundary conditions, where $\bar{E}$ is the unknown electric field inside the sphere, $k$ is the free-space wave number, and $\mathrm{m}$ is the refractive index defined as

$$
\mathrm{m}^{2}=\epsilon_{\mathrm{r}}-\mathrm{j} \sigma /\left(\omega \epsilon_{0}\right)
$$

with $\epsilon_{r}$ as the dielectric constant (permittivity relative to the free-space value $\epsilon_{0}$ ) and $\sigma$ the conductivity of the material. In free space, $\epsilon_{r}=1, \sigma$ $=0$, and $\mathrm{m}=1$. We use $\mathrm{m}$ for refractive index to be consistent with common notation [7], but this $m$ should not be confused with the impedance mismatch factor in eq (16).

To solve the vector wave equation we have to obtain solutions to the corresponding scalar wave equation,

$$
\nabla^{2} \mathrm{u}+\mathrm{k}^{2} \mathrm{~m}^{2} \mathrm{u}=0
$$

in spherical coordinates. Since eq (A3) is a second-order partial differential equation, we have two independent solutions, $u_{1}$ and $u_{2}$ [7].

Let us represent the incident wave outside the sphere where $\mathrm{m}=1$ as

$$
\begin{aligned}
& u_{1}=\cos \phi \sum_{n=1}^{\infty}(-j)^{n} \frac{2 n+1}{n(n+1)} P_{n}^{1}(\cos \theta) j_{n}(k r), \\
& u_{2}=\sin \sum_{n=1}^{\infty}(-j)^{n} \frac{2 n+1}{n(n+1)} P_{n}^{1}(\cos \theta) j_{n}(k r),
\end{aligned}
$$

where $(\phi, \theta, r)$ are the spherical coordinates with $r=0$ at the sphere center, $\mathrm{P}_{\mathrm{n}}^{1}$ is the associate Legendre function, and $j_{\mathrm{n}}$ is the spherical Bessel function.

The scattered wave outside the sphere can then be expressed as 


$$
\begin{aligned}
& u_{i}^{\prime}=\cos \phi \sum_{n=1}^{\infty}\left(-a_{n}\right)(-j)^{n} \frac{2 n+1}{n(n+1)} P_{n}^{1}(\cos \theta) h_{n}^{(2)}(k r), \\
& u_{2}^{\prime}=\sin \phi \sum_{n=1}^{\infty}\left(-b_{n}\right)(-j)^{n} \frac{2 n+1}{n(n+1)} P_{n}^{1}(\cos \theta) h_{n}^{(2)}(k r),
\end{aligned}
$$

where $h_{n}^{(2)}$ is the spherical Hankel function, and $a_{n}$ and $b_{n}$ are the unknown coefficients to be determined.

The wave penetrated into the sphere where $m$ is complex may be written as

$$
\begin{aligned}
& u_{1}^{\prime \prime}=\cos \phi \sum_{n=1}^{\infty}\left(m c_{n}\right)(-j)^{n} \frac{2 n+1}{n(n+1)} P_{n}^{1}(\cos \theta) j_{n}(m k r), \\
& u_{2}^{\prime \prime}=\sin \phi \sum_{n=1}^{\infty}\left(m d_{n}\right)(-j)^{n} \frac{2 n+1}{n(n+1)} P_{n}^{1}(\cos \theta) j_{n}(m k r),
\end{aligned}
$$

where $c_{n}$ and $d_{n}$ are another unknown coefficients to be determined.

The boundary condition requires that at the sphere surface $r=a$,

$$
u_{1}+u_{1}^{\prime}=u_{1}^{\prime \prime} \text {, and } u_{2}+u_{2}^{\prime}=u_{2}^{\prime \prime} \text {, }
$$

from which we obtain, after some algebraic simplifications,

$$
a_{n}=A / B, \quad b_{n}=C / D, \quad c_{n}=j / B, \quad \text { and } d_{n}=j / D \text {, }
$$

where

$$
A=\Psi_{n}^{\prime}(y) \Psi_{n}(x)-m \Psi_{n}(y) \Psi_{n}^{\prime}(x)
$$




$$
\begin{aligned}
& B=\Psi_{n}^{\prime}(y) \xi_{n}(x)-m \Psi_{n}(y) \xi_{n}^{\prime}(x), \\
& C=m \Psi_{n}^{\prime}(y) \Psi_{n}(x)-\Psi_{n}(y) \Psi_{n}^{\prime}(x), \\
& D=m \Psi_{n}^{\prime}(y) \xi_{n}(x)-\Psi_{n}(y) \xi_{n}^{\prime}(x),
\end{aligned}
$$

with $\mathrm{x}=\mathrm{ka}$, and $\mathrm{y}=\mathrm{mka}=\mathrm{mx}$.

In eq (A9), $\Psi_{n}$ and $\xi_{n}=\Psi_{n}+j \chi_{n}$ are the Riccati-Bessel functions [7], and $\Psi_{\mathrm{n}}^{\prime}$ and $\xi_{\mathrm{n}}^{\prime}$ are the derivatives with respect to their arguments.

Fortunately, the spherical and Riccati Bessel functions can be written more specifically when $\mathrm{n}$ is an integer, which is the case in our study. The first few lower orders of them are given below.

Spherical Bessel functions:

$$
\begin{aligned}
& j_{0}(z)=z^{-1} \sin z \\
& j_{1}(z)=z^{-2} \sin z-z^{-1} \cos z \\
& j_{2}(z)=\left(3 z^{-3}-z^{-1}\right) \sin z-3 z^{-2} \cos z, \\
& j_{3}(z)=\left(15 z^{-4}-6 z^{-2}\right) \sin z^{-\left(15 z^{-3}-z^{-1}\right) \cos z,} \\
& j_{4}(z)=\left(105 z^{-5}-45 z^{-3}+z^{-1}\right) \sin z-\left(105 z^{-4}-10 z^{-2}\right) \cos z .
\end{aligned}
$$

For still higher orders, they can be deduced from the recursion formula, 


$$
j_{n}(z)=f_{n}(z) \sin z+(-1)^{n+1} f_{-n-1}(z) \cos z
$$

and

$$
f_{n-1}(z)+f_{n+1}(z)=(2 n+1) z^{-1} f_{n}(z),
$$

with $\quad f_{0}=z^{-1}, \quad f_{1}=z^{-2}, \quad f_{-1}=0, \quad$ and $\quad f_{-2}=-z^{-1}$.

In eqs $(A 10)$ and $(A 11)$, as $z \rightarrow 0, j_{0}(z) \rightarrow 1$, and $j_{n}(z) \rightarrow 0$ for $n \neq 0$.

Riccati-Bessel functions:

$$
\begin{aligned}
& \Psi_{0}(z)=\sin z \\
& \Psi_{1}(z)=z^{-1} \sin z-\cos z \\
& \Psi_{2}(z)=\left(3 z^{-2}-1\right) \sin z-3 z^{-1} \cos z \\
& \Psi_{3}(z)=\left(15 z^{-3}-6 z^{-1}\right) \sin z-\left(15 z^{-2}-1\right) \cos z, \\
& \Psi_{4}(z)=\left(105 z^{-4}-45 z^{-2}+1\right) \sin z-\left(105 z^{-3}-10 z^{-1}\right) \cos z ; \\
& \chi_{0}(z)=\cos z \\
& \chi_{1}(z)=\sin z+z^{-1} \cos z, \\
& \chi_{2}(z)=3 z^{-1} \sin z+\left(3 z^{-2}-1\right) \cos z
\end{aligned}
$$




$$
\chi_{3}(z)=\left(15 z^{-2}-1\right) \sin z+\left(15 z^{-3}-6 z^{-1}\right) \cos z
$$

and

$$
\chi_{4}(z)=\left(105 z^{-3}-10 z^{-1}\right) \sin z+\left(105 z^{-4}-45 z^{-2}+1\right) \cos z
$$

The derivatives of the Riccati-Bessel functions required in (A9) are then

$$
\begin{aligned}
& \Psi_{0}^{\prime}(z)=\cos z \\
& \Psi_{1}^{\prime}(z)=\left(-z^{-2}+1\right) \sin z^{-1} \cos z \\
& \Psi_{2}^{\prime}(z)=\left(-6 z^{-3}+3 z^{-1}\right) \sin z+\left(6 z^{-2}-1\right) \cos z \\
& \Psi_{3}^{\prime}(z)=\left(-45 z^{-4}+21 z^{-2}-1\right) \sin z+\left(45 z^{-3}-6 z^{-1}\right) \cos z \\
& \Psi_{4}^{\prime}(z)=\left(-420 z^{-5}+195 z^{-3}-10 z^{-1}\right) \sin z+\left(420 z^{-4}-55 z^{-2}+1\right) \cos z \\
& \chi_{0}^{\prime}(z)=-\sin z \\
& \chi_{3}^{\prime}(z)=\left(-45 z^{-3}+6 z^{-1}\right) \sin z+\left(-45 z^{-4}+21 z^{-2}-1\right) \cos z \\
& \chi_{2}^{\prime}(z)=\left(-6 z^{-2}+1\right) \sin z+\left(-6 z^{-3}+3 z^{-1}\right) \cos z \\
& \chi_{1}^{-1} \sin z+\left(-z^{-2}+1\right) \cos z
\end{aligned}
$$

and

$$
\chi_{4}^{\prime}(z)=\left(420 z^{-4}+55 z^{-2}-1\right) \sin z+\left(-420 z^{-5}+195 z^{-3}-10 z^{-1}\right) \cos z
$$


In eqs (A13) and (Al4), $z=x$ (real) or $z=y$ (complex). Once the frequency $f$ (thus $\omega$ ), sphere radius a, and material constants $\epsilon_{\mathrm{r}}$ and $\sigma$ are specified, the coefficients $a_{n}, b_{n}, c_{n}$, and $d_{n}$ can be computed from eqs (A8) and (A9), and the field distributions inside and outside the sphere determined from eqs (A4), (A5), and (A6). The number of terms required in eqs (A4) to (A6) depends on ka.

Two other efficiency factors, the total factor $\eta_{t}$ and the scattered factor $\eta_{s}$, are needed. They are defined by

$$
\eta_{t}=2(k a)^{-2} \sum_{n=1}^{\infty}(2 n+1) \operatorname{Re}\left(a_{n}+b_{n}\right)
$$

and

$$
\eta_{s}=2(k a)^{-2} \sum_{n=1}^{\infty}(2 n+1)\left[\left|a_{n}\right|^{2}+\left|b_{n}\right|^{2}\right]
$$

The efficiency factor $\eta_{a}$ for absorption is then

$$
\eta_{\mathrm{a}}=\eta_{\mathrm{t}}-\eta_{\mathrm{s}}
$$

The absorption area of the sphere is obtained from $\sigma_{a}=\left(\pi a^{2}\right) \eta_{a}$, which in turn is used to compute the power loss due to absorption.

When ka becomes very large, this theory is not convenient because the summations converge too slowly. In this case, a geometrical optics approximation $[7, \mathrm{Sec}, 14.23]$ can be used to compute $\sigma_{a}$. The computer program in Appendix $F$ uses this approximation when ka becomes large. Consequently, the $\mathrm{Q}_{2}$ curve can show a small discontinuity when the frequency reaches the crossover value (at $\mathrm{ka}=46.0$ ). 
APPENDIX B. TRANSMISSION CROSS SECTION OF A SMALL CIRCULAR APERTURE

Consider a small circular aperture $(k a<1)$ in a planar sheet as shown in figure 3. The transmitted fields can be written as the fields of a tangential magnetic dipole moment $\mathrm{p}_{\mathrm{m}}$ and a normal electric dipole moment $\mathrm{p}_{\mathrm{e}}$ that can be written as the product of an aperture polarizability times the appropriate incident field $[8,13]$ :

$$
\mathrm{P}_{\mathrm{m}}=\alpha_{\mathrm{m}} \mathrm{H}_{\tan }^{\mathrm{Sc}} \text { and } \mathrm{P}_{\mathrm{e}}=\epsilon_{0} \alpha_{\mathrm{e}} \mathrm{E}_{\mathrm{n}}^{\mathrm{Sc}}
$$

where $\mathrm{H}_{\text {tan }}^{\mathrm{Sc}}$ is the tangential magnetic field at the center of the shortcircuited aperture and $\mathrm{E}_{\mathrm{n}}^{\mathrm{SC}}$ is the normal electric field at the center of the short-circuited aperture. The magnetic and electric polarizabilities, $\alpha_{\mathrm{m}}$ and $\alpha_{e}$, are given by $[8,13]$

$$
\alpha_{m}=4 a^{3} / 3 \text { and } \alpha_{e}=2 a^{3} / 3
$$

The dipole moments radiate in the presence of the ground plane (so their images are included), and the total transmitted power (radiated into one half-space) is [26]

$$
\mathrm{P}_{t}=\frac{4 \pi \eta_{0}}{3 \lambda^{2}}\left(\mathrm{k}^{2}\left|\mathrm{p}_{\mathrm{m}}\right|^{2}+\left|\mathrm{p}_{\mathrm{e}}\right|^{2}\right)
$$

We consider the cases of parallel and perpendicular polarizations separately. For parallel polarization, the short-circuited fields are

$$
\mathrm{H}_{\tan }^{\mathrm{SC}}=2 \mathrm{H}_{i} \text { and } \mathrm{E}_{\mathrm{n}}^{\mathrm{SC}}=2 \mathrm{E}_{\mathrm{i}} \sin \theta^{\mathrm{i}}
$$

where the incident fields can be related to the incident power density $s_{i}$ by 


$$
S_{i}=\eta_{0} H_{i}^{2} \text { and } s_{i}=E_{i}^{2} / \eta_{0} .
$$

From eqs (B1)-(B5), we can write the transmission cross section for paralle1 polarization as

$$
\sigma_{\text {tpar }}=P_{t} / S_{i}=\frac{64}{27 \pi} k^{4} a^{6}\left(1+\frac{1}{4} \sin ^{2} \theta^{i}\right),
$$

which is the result needed in Section 3.2.

For perpendicular polarization, the short-circuited fields are

$$
\mathrm{H}_{\tan }^{\mathrm{Sc}}=2 \mathrm{H}_{\mathrm{i}} \cos \theta^{i} \text { and } \mathrm{E}_{\mathrm{n}}^{\mathrm{Sc}}=0 .
$$

From eqs (B1)-(B3), (B5), and (B7), we can write the transmission cross section for perpendicular polarization as

$$
\sigma_{\text {tperp }}=\frac{64}{27 \pi} k^{4} a^{6} \cos ^{2} \theta^{i},
$$

which is the other result needed in Section 3.2.

\section{APPENDIX C. RADAR CHARACTERISTICS}

Four types of radars that are likely to be encountered in the vicinity of commercial U.S. airports. A summary of these is given below.

A. FAA ARSR very long range search radar. This radar system operates on fixed frequencies in the 1250-1300 $\mathrm{MHz}$ band with a peak power of $4 \mathrm{MW}$. The pulse duration is $2-5 \mu \mathrm{s}$ with a pulse repetition rate of $300 \mathrm{pulses} / \mathrm{s}$ and a rise time of approximately 108 of the pulse width. The radar antenna completes one revolution every $10 \mathrm{~s}$. This is a moving target indicator (MTI) radar that uses a pulse stagger sequence in order to avoid blind speeds. 
B. ASR airport search radar. This radar is used for air traffic control in the immediate vicinity of commercial airports. This system operates in the 2700-2900 MHz band with a peak power of $850 \mathrm{~kW}$. The pulse width is $0.8 \mu \mathrm{s}$ with a pulse repetition rate of 1000 pulses/s and a rise time of 108 of the pulse width. The antenna rotates every $4.75 \mathrm{~s}$. This is an MTI radar with no stagger sequence.

C. National Weather Service TDWR radar. This is a Doppler weather radar that operates in the $5600 \mathrm{MHz}$ band with a peak power of $800 \mathrm{~kW}$. The pulse width is $3 \mu \mathrm{s}$ with a pulse repetition rate of 1000 pulses/s. The antenna can operate in either a fixed mode (to look at interesting weather) or rotate once every $20 \mathrm{~s}$. There is no staggering in the pulse sequence.

D. ASDE ground radar. This system is used to monitor ground terminal traffic at many airports (such as parked and taxiing aircraft). This system operates in the $22 \mathrm{GHz}$ band with a peak power of $1 \mathrm{~kW}$, with a pulse width of 50-60 ns and a pulse repetition rate of 10000 pulses/s.

APPENDIX D. SCALING

For applications involving large objects, such as aircraft, laboratory measurements are more conveniently done on smaller scale models. For the time-harmonic form of Maxwell's equations, scaling of frequency and length in nondispersive, lossless media is well known. For the example of frequency-independent antennas [27], "the entire electrical performance is frequency-independent if all length dimensions are scaled in inverse proportion to frequency."

To consider the more general case of lossy media, we begin with the time-harmonic, source-free form of Maxwell's equations for $\exp (j \omega t)$ time dependence:

$$
\nabla \times \overline{\mathrm{H}}(\overline{\mathrm{r}}, \omega)=[j \omega \epsilon(\overline{\mathrm{r}})+\sigma(\overline{\mathrm{r}})] \overline{\mathrm{E}}(\overline{\mathrm{r}}, \omega),
$$




$$
\nabla \times \overline{\mathrm{E}}(\overline{\mathrm{r}}, \omega)=-j \omega \mu(\overline{\mathrm{r}}) \overline{\mathrm{H}}(\overline{\mathrm{r}}, \omega)
$$

where the magnetic permeability $\mu$, the permittivity $\epsilon$, and the conductivity $\sigma$ are assumed to be independent of frequency, but can be functions of position $\bar{r}$. Suppose that we wish to scale (multiply) lengths by a real factor $1 / \mathrm{s}$ (that can be greater than or less than 1 ):

$$
\bar{r}^{\prime}=\bar{r} / s \text { or } \bar{r}=s \bar{r}^{\prime} \text {. }
$$

If $s>1$, then the new primed lengths are less than the original lengths.

To examine the scaling possibilities of eq (D1), we rewrite the del ( $\nabla)$ operator:

$$
\nabla=\bar{u}_{x} \frac{\partial}{\partial x}+\bar{u}_{y} \frac{\partial}{\partial y}+\bar{u}_{z} \frac{\partial}{\partial z}=\frac{1}{s}\left(\bar{u}_{x} \frac{\partial}{\partial x^{\prime}}+\bar{u}_{y} \frac{\partial}{\partial y^{\prime}}+\bar{u}_{z} \frac{\partial}{\partial z^{\prime}}\right)=\frac{1}{s} \nabla^{\prime},
$$

where $\bar{u}_{x}, \quad \bar{u}_{y}, \quad$ and $\bar{u}_{z}$ are unit vectors that remain unchanged in the primed coordinate system. If we substitute eq (D3) into eq (D1) and multiply by $s$, then we have

$$
\begin{aligned}
& \nabla^{\prime} \times \overline{\mathrm{H}}\left(s \overline{\mathrm{r}}^{\prime}\right)=\left[j \omega s \epsilon\left(s \overline{\mathrm{r}}^{\prime}\right)+s \sigma\left(s \overline{\mathrm{r}}^{\prime}\right)\right] \overline{\mathrm{E}}\left(s \mathrm{r}^{\prime}\right), \\
& \nabla^{\prime} \times \overline{\mathrm{E}}\left(s \overline{\mathrm{r}}^{\prime}\right)=-j \omega s \mu\left(s \overline{\mathrm{r}}^{\prime}\right) \overline{\mathrm{H}}\left(s \overline{\mathrm{r}}^{\prime}\right) .
\end{aligned}
$$

The object now is to scale quantities on the right side of eq (D4) to bring it to the same form given in eq (D1). There are two possibilities: (1) we could scale $\epsilon, \sigma$, and $\mu$ by $s$ or (2) we could scale $\omega$ and $\sigma$ by $s$. The first possibility is of no value for scaled experiments. The second possibility is the standard length/frequency scaling for lossless media where $\sigma$ is either 0 or $\infty$. For those cases, scaling of $\sigma$ has no effect on the results. We choose the second possibility and the following specific scaling: 


$$
\overline{\mathrm{H}}^{\prime}\left(\overline{\mathrm{r}}^{\prime}\right)=\overline{\mathrm{H}}\left(\mathrm{s} \overline{\mathrm{r}}^{\prime}\right), \overline{\mathrm{E}}^{\prime}\left(\overline{\mathrm{r}}^{\prime}\right)=\overline{\mathrm{E}}\left(\mathrm{s} \overline{\mathrm{r}}^{\prime}\right), \overline{\mathrm{r}}^{\prime}=\frac{1}{\mathrm{~s}} \overline{\mathrm{r}},
$$

and

$$
\omega^{\prime}=s \omega, \sigma^{\prime}\left(\bar{r}^{\prime}\right)=s \sigma\left(s \bar{r}^{\prime}\right), \epsilon^{\prime}\left(\bar{r}^{\prime}\right)=\epsilon\left(s \bar{r}^{\prime}\right), \mu^{\prime}\left(\bar{r}^{\prime}\right)=\mu\left(s \bar{r}^{\prime}\right) .
$$

If we substitute eq (D5) into eq (D1), we obtain

$$
\begin{aligned}
& \nabla^{\prime} \times \overline{\mathrm{H}}^{\prime}\left(\overline{\mathrm{r}}^{\prime}, \omega^{\prime}\right)=\left[\mathrm{j} \omega^{\prime} \epsilon^{\prime}\left(\overline{\mathrm{r}}^{\prime}\right)+\sigma^{\prime}\left(\overline{\mathrm{r}}^{\prime}\right)\right] \overline{\mathrm{E}}^{\prime}\left(\overline{\left.\mathrm{r}^{\prime}, \omega^{\prime}\right),}\right. \\
& \nabla^{\prime} \times \overline{\mathrm{E}}^{\prime}\left(\overline{\mathrm{r}}^{\prime}, \omega^{\prime}\right)=-\mathrm{j} \omega^{\prime} \mu^{\prime}\left(\overline{\mathrm{r}}^{\prime}\right) \overline{\mathrm{H}}\left(\overline{\mathrm{r}}^{\prime}, \omega^{\prime}\right) .
\end{aligned}
$$

Equations (D6) are identical to Maxwell's eqs (D1) except that all quantities are primed. So they are equivalent under the scaling transformations in eq (D5). To summarize, we scale all distances by $1 / s$, frequency by $s$, and conductivity by $s$. If we wish to perform a reduced-size $(s>1)$ scale model experiment, then we increase frequency by a factor $s$ as expected, but we also need to increase conductivity by a factor $s$. This obviously presents a materials problem, but some ways around this problem are discussed in the remainder of this section.

The required conductivity scaling can be explained in an equivalent manner. The first eq (D1) can also be written

$$
\nabla \times \overline{\mathrm{H}}(\overline{\mathrm{r}}, \omega)=j \omega \epsilon_{\mathrm{c}}(\overline{\mathrm{r}}) \overline{\mathrm{E}}(\overline{\mathrm{r}}, \omega),
$$

where

$$
\epsilon_{c}(\bar{r})=\epsilon_{r}(\bar{r})-j \sigma(\bar{r}) / \omega .
$$

Since our frequency scaling requires multiplying $\omega$ by $s$, we must also multiply $\sigma$ by $s$ to keep the imaginary part of the complex dielectric constant $\epsilon_{c}$ from changing.

For general cavity applications, if the conductivity is not scaled according to eq (D5), the field distributions will change and the resonant frequencies and $Q$ will also change in an unpredictable manner. However, if the walls are highly conducting, then the resonant frequencies will not 
depend on the wall conductivity and will scale as s times the resonant frequencies of the original cavity.

Consider now the $Q$ of a cavity with highly conducting walls. We examine the individual $Q$ s separately as in Section 2. The expression for $Q_{1}$ is given by eq ( 8 ). For the scaling in eq (D5), the new primed quantities are

$$
Q_{1}^{\prime}=\frac{3 V^{\prime}}{2 \mu_{r} S^{\prime} \delta^{\prime}}=Q_{1} \text {, where } V^{\prime}=\frac{V}{s^{3}}, S^{\prime}=\frac{S}{s^{2}}, \delta^{\prime}=\frac{\delta}{s^{\prime}}, \omega^{\prime}=s \omega
$$

and $\sigma^{\prime}=s \sigma$. If it is not possible to scale wall conductivity, then we have

$$
Q_{1}^{\prime}=Q_{1} s^{-1 / 2}, \delta^{\prime}=\delta s^{-1 / 2} \text {, and } \sigma^{\prime}=\sigma
$$

Thus the $Q_{1}^{\prime}$ of the scaled cavity will decrease if the frequency is scaled up ( $s>1$ ), lengths are scaled down, and wall conductivity is not scaled. Consider now $\mathrm{Q}_{2}$. If the loading objects have high conductivity (as for metal), then $Q_{2}^{\prime}$ will change by the factor $s^{-1 / 2}$ as in eq (D10) because the loading objects have the same loss dependence on frequency and conductivity as the walls. A different situation arises when the cavity losses are primarily due to objects of low conductivity and low permittivity (nonmetal objects). In this case the Born approximation [28] states that the field distribution is not strongly affected by the loading objects. Thus the resonant frequencies are not significantly changed, and the cavity loss is proportional to the conductivity $\sigma$ of the loading objects. Then $Q_{2}$ is inversely proportional to the conductivity of loading objects:

$$
\mathrm{Q}_{2} \propto \omega / \sigma
$$

If we scale frequency, length, and conductivity, then the new $Q_{2}^{\prime}$ is 


$$
\mathrm{Q}_{2}^{\prime}=\mathrm{Q}_{2} \propto \omega^{\prime} / \sigma^{\prime} \text {, where } \omega^{\prime}=\mathrm{s} \omega \text { and } \sigma^{\prime}=\mathrm{s} \sigma \text {. }
$$

If we are not able to scale conductivity, then

$$
Q_{2}^{\prime}=s Q_{2} \text {, where } \omega^{\prime}=s \omega \text { and } \sigma^{\prime}=\sigma \text {. }
$$

Here the change in $Q^{\prime}$ is in the opposite direction as that in eq (D10) where the wall conductivity is unscaled. The actual situation for low conductivity objects (such as people and nonmetal furniture) is that the unscaled conductivity is equal to frequency times the imaginary part of the dielectric constant:

$$
\sigma=\omega \operatorname{Im}\left(\epsilon_{c}\right)
$$

If Im $\left(\epsilon_{c}\right)$ does not change with frequency, then the proper conductivity scaling automatically occurs with no change in loading material.

Consider now $Q_{3}$ and $Q_{4}$. For aperture leakage losses and antenna reception in a fixed load impedance, length and frequency scaling are sufficient to maintain the $\mathrm{Q}_{3}$ and $\mathrm{Q}_{4}$ with no change upon scaling.

Thus we have three situations with regard to cavity scaling where frequency is scaled up $(s>1)$, length is inversely scaled, and conductivity is unscaled. For dominant wall losses, the $Q^{\prime}$ drops by a factor $s^{-1 / 2}$ as given by eq (D10). For dominant loading losses due to low conductivity, the $Q^{\prime}$ increases by a factor $s$ as given by eq (D13). (This change will be less or even zero for dielectrics with a nearly constant loss tangent.) For dominant aperture leakage or antenna reception losses, the $Q^{\prime}$ is unchanged.

In summary, the resonant frequencies will scale with $s$ if the field distributions change little. The cavity $Q^{\prime}$ can be higher or lower than the original $Q$ if the conductivity is unscaled. However, the magnitude and direction of change can be predicted if we know the dominant loss mechanism. 
APPENDIX E. ELECTRICAL PROPERTIES OF SALT WATER

The electrical properties of salt water have been investigated by Saxton and Lane [18] as a function of frequency, salt concentration, and temperature. The relative complex dielectric constant $\epsilon_{\mathrm{cr}}$ can be approximated by a combination of Debye relaxation and dc conductivity:

$$
\epsilon_{c r}=\frac{\epsilon_{s}-\epsilon_{h}}{1+\omega^{2} t_{r}^{2}}+\epsilon_{h}-j\left[\frac{\sigma}{\omega \epsilon_{0}}+\frac{\left(\epsilon_{s}-\epsilon_{h}\right) \omega t_{r}}{1+\omega^{2} t_{r}^{2}}\right],
$$

where $\omega$ is the angular frequency and $\epsilon_{0}$ is the permittivity of free space. The remaining parameters that characterize salt water are: static dielectric $\epsilon_{s}$, high-frequency dielectric constant $\epsilon_{h}$, dielectric relaxation time $t_{r}$, and dc conductivity $\sigma$.

The real part of the relative dielectric constant varies from $\epsilon_{s}$ at low frequencies to $\epsilon_{\mathrm{h}}$ at high frequencies. The imaginary part consists of two terms that represent conduction loss and dielectric relaxation loss. The dielectric relaxation loss reaches its maximum at $\omega=1 / t_{r}$. To represent sea water at room temperature $\left(20^{\circ} \mathrm{C}\right)$, we select the following values for the parameters [18]: $\epsilon_{\mathrm{s}}=70.0, \epsilon_{\mathrm{h}}=4.9, t_{\mathrm{r}}=9.2 \times 10^{-12} \mathrm{~s}$, and $\sigma=4.0 \mathrm{~s} / \mathrm{m}$. The results from eq (E1) using these parameters are consistent with more recent investigations of electrolytic solutions [29].

APPENDIX F. SOFTWARE TO ANALYZE A RECTANGULAR CAVITY

We have written a computer program to analyze a rectangular cavity based on the theory in Sections 2-4. This program was used to provide the theoretical results for comparison with measured data in Section 5 . This software may not be the ultimate form for application software, but potential users may find it to be very helpful. We are therefore including the source code in this appendix, along with instructions on how to use it. 
The program is written in Fortran 77 and can be compiled to run on whatever computer is available. This includes personal computers with a 386 processor and 387 coprocessor or $486 \mathrm{DX}$ processor.

Input data

The compiled program will look for an input data file of whatever name is selected by the input symbol ' $<$ '. For example, run 'DFAA7 < $N 11^{\prime}$ where DFAA7 is the executable file and N1l is the input data file.

An example of the input file is shown before the source code. All input data should be in SI units. The use of ' $D$ ' rather than ' $E$ ' for exponents indicates double precision numbers.

On the first two lines, the numbers under $A, B$, and $C$ are the dimensions of the rectangular-shaped cavity. The number under WCOND is the wall conductivity, the number under RMU is the relative permeability of the walls, the number under NANT is the number of antennas inside the cavity that couple energy out of the cavity, and the number under NAPRAD is the number of different sizes of apertures.

On the third line, the column headers APRAD and NAP refer to aperture radius and number of apertures, respectively. If there are no apertures, both NAPRAD and all NAP values should be 0 . Up to six different aperture sizes can be used.

On lines 10 and 11 , the number under NHSPH is the number of absorptive spheres with salt water. Salt water provides a good approximation to the absorption of people. The number under HSPRAD is the radius of these spheres. The number under HCNDSP is the conductivity of ocean salt water. The number under EPSLP is the low-frequency relative dielectric constant of salt water. The number under EPSHP is the high-frequency dielectric constant of salt water. The number under TR is the dielectric relaxation time. See Appendix E. Only the first two numbers should be changed by a program user.

On lines 12 and 13, the number of spheres of other material may be treated. No frequency dependence is allowed. The number under NOSPH is the number of spheres, and the number under OSPRAD is the radius of these 
spheres. The number under OCONDSP is the conductivity of the sphere material, and the number under EPSOP is its relative dielectric constant.

On lines 14 and 15, the number under FREQL is the start frequency, the number under DF is the frequency increment, and the number under NF is the number of frequency steps.

\section{Output data}

The output data are always put into a file 'CAVO.CSV'. The CSV is a comma-separated variable format for use with a spread sheet and for plotting. This format could be changed to any other desired format.

The first 15 lines repeat the input data and also give the volume and surface area of the cavity.

The output is in 15 columns with the data within each row identified by a column header.

These are: frequency, transmission cross-section average, Q1 (Q due to wall loss), Q2H (Q due to humanoid absorption), Q20 (Q due to other absorptive materials), Q3(1) (Q due to first size of aperture), Q3(2) (Q due to second size of aperture), ... Q3(6) (Q due to sixth size of aperture), Q4 ( $Q$ due to antenna coupling), Q (composite of all Qs), TAU (time constant), and SE (shielding effectiveness).

A spread sheet or plotting program is very helpful in displaying these data for insights and interpretations.

Input file:

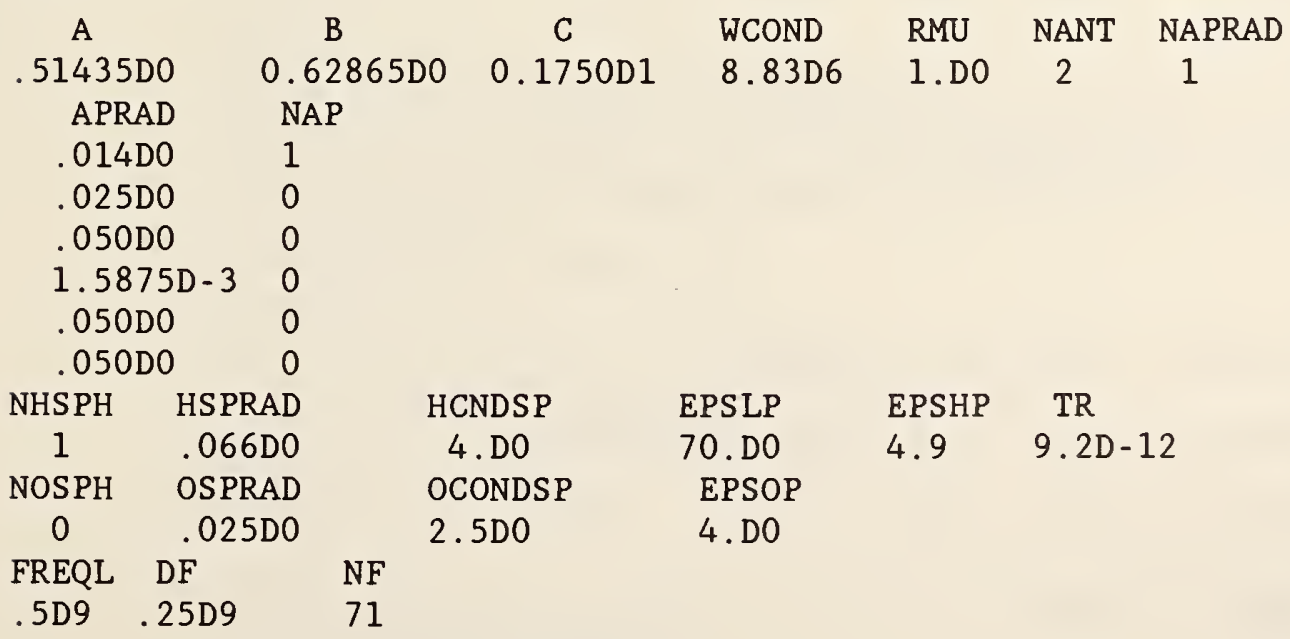


Source code:

\section{PROGRAM DFAA7}

C THIS PROGRAM IS BASED ON THE WORK OF DAVID HILL AND MARK MA OF

C NIST, BOULDER, CO. THE THEORY WAS DEVELOPED DURING LATE 1992.

C THE PROGRAM WAS WRITTEN JAN. 93 BY DAVID HILL, 303-497-3472.

C IT WAS MODIFIED FEB 93 FOR USE ON A 386 OR 486 PC

C BY ABBIE O'GALLAGHER, 303-497-3266.

C THIS DEVELOPMENT WAS SUPPORTED BY THE FAA TO SUPPORT HIRF TESTING

C REQUIREMENTS, BUT IS BROADLY APPLICABLE TO RESONANT CAVITIES.

C THERE WILL NOW BE 2 SETS OF DATA HAVING TO DO WITH SPHERES. THE ONES

C THAT START WITH H HAVE TO DO WITH HUMANOID (SALT-WATER) SPHERES. NHSPH

C IS THE NUMBER OF HUMANOID SPHERES.

C Q2H IS THE ENERGY LOSS DUE TO HUMANOID SHPERES.

C QWO WILL BE THE ENERGY LOSS DUE TO OTHER KINDS OF SHERES.

C NOSPH,OSPRAD, OCNDSP AND EPSOP HAVE TO DO WITH OTHER KINDS OF SPHERES. INTEGER MAXNAR PARAMETER (MAXNAR=6)

C THE PARAMETER NAPRAD IS THE NUMBER OF DIFFERENT APERTURE RADII

C MAXNAR IS THE (ARBITRARILLY) MAXIMUM VALUE NAPRAD CAN TAKE DOUBLE PRECISION PI, EPSO, PERMO, RMU , CL, A , B , C , APRAD (MAXNAR), 1 WCOND, HSPRAD, TEMP,

1 HCNDSP , EPSP , V , S , EPSLP , EPSHP , TR , X , DEN ,

1 FREQL, DF , FREQ, FW , DEL, Q1, WL, WK, EPS I , Q2H, S IGABS ;

1 SIGAVE (MAXNAR), TS IGAV, Q3 (MAXNAR), Q4, TAU, Q, SE , PDRAT , A,

1 OS PRAD , OCNDSP , EPSOP, Q2O

INTEGER NAP (MAXNAR), NANT , NHSPH , NF , I , NT , NOS PH, NAPRAD , J

DOUBLE COMPLEX EPSC, REFI

OPEN ( 3, FILE $=$ ' CAVO. CSV')

REWIND 3

$\mathrm{PI}=\mathrm{DACOS}(-1$. D0 $)$

5 FORMAT (1X)

DO $1 \quad I=1$, MAXNAR

$\operatorname{NAP}(I)=0$

1 CONTINUE

$\mathrm{EPSO}=8.854 \mathrm{D}-12$

PERMO $=P I * 4 . D-7$

$\mathrm{CL}=3$. $\mathrm{D} 8$

$\operatorname{READ}(*, 5)$

$\operatorname{READ}(*, *) A, B, C$, WCOND , RMU , NANT , NAPRAD

IF (NAPRAD .GT . MAXNAR) THEN

PRINT*,'ERROR. MAXNAR SMALLER THAN NAPRAD'

STOP

ENDIF

$\operatorname{READ}(*, 5)$

$\operatorname{READ}(*, *)$ (APRAD $(I), \operatorname{NAP}(I), I=1, \operatorname{MAXNAR})$

$\operatorname{READ}(*, 5)$

$\operatorname{READ}(*, *)$ NHSPH, HSPRAD, HCNDSP, EPSLP, EPSHP , TR

$\operatorname{READ}(*, 5)$

$\operatorname{READ}(*, *)$ NOSPH, OSPRAD, OCNDSP, EPSOP

$\operatorname{READ}(*, 5)$

$\operatorname{READ}(*, *)$ FREQL, DF , NF

WRITE $(3,10)$ 
10 FORMAT (' A, B, C , WALLCOND, RELMU, NAPRAD')

WRITE $(3,20)$ A, B, C, WCOND, RMU, NAPRAD

20 FORMAT ( 5 (E15.5,', '), I10)

WRITE $(3,11)$

11 FORMAT (' APRAD, NAP') WRITE $(3,31)$ (APRAD (I), NAP(I),$I=1$, MAXNAR)

31 FORMAT (E15.5,', ', I10)

WRITE $(3,23)$

23 FORMAT (' NANT, NHSPH, HSPHERERAD, HSPHERECOND, SEPSL, SEPSH, RELTIME' )

WRITE $(3,27)$ NANT , NHSPH, HS PRAD , HCNDSP , EPSLP , EPSHP , TR

27 FORMAT (2(I10,', ') ,4(E15.5,', ' ), E15.5)

WRITE $(3,28)$

28 FORMAT (' NOSPH, SPHERERAD, SPHERECOND, SPHEREPS , ')

WRITE $(3,29)$ NOSPH, OSPRAD, OCNDSP, EPSOP

29 FORMAT $\left(\left(\mathrm{I} 10,^{\prime},,^{\prime}\right), 2\left(\mathrm{E} 15.5,^{\prime},,^{\prime}\right)\right.$, E15.5)

$\mathrm{V}=\mathrm{A} * \mathrm{~B} * \mathrm{C}$

C

C

C

C

C

C

APPLY CORRECTION TO VOLUME FROM BARE CAVITY TO COMPENSATE FOR VOLUME OF ENCLOSED STRUCTURES

$\mathrm{V}=\mathrm{V} *$. 90D0

$\mathrm{S}=2 . \mathrm{D} 0 *(\mathrm{~A} * \mathrm{~B}+\mathrm{A} * \mathrm{C}+\mathrm{B} * \mathrm{C})$

APPLY CORRECTION TO SURFACE OF BARE CAVITY TO COMPENSATE FOR SURFACE AREA OF ENCLOSED STRUCTURES

$\mathrm{S}=\mathrm{S} * 1.2 \mathrm{DO}$

WRITE $(3,30)$

30 FORMAT (' VOL, SURF')

WRITE $(3,40) \mathrm{V}, \mathrm{S}$

40 FORMAT (E15.5,', ', E15.5)

WRITE $(3,50)$

50 FORMAT (' FREQ, SIGAVE, Q1, Q2H, Q20,Q3(1), Q3 (2), Q3 (3), Q3 (4), Q3 (5),'

1

DO $60 \mathrm{I}=1, \mathrm{NF}$ , 'Q3(6), $\mathrm{Q} 4, \mathrm{Q}, \mathrm{TAU}, \mathrm{SE}$ ')

FREQ $=$ FREQL $+(I-1) * D F$

$F W=2 . D 0 * P I * F R E Q$

$\mathrm{DEL}=\mathrm{SQRT}(2 . \mathrm{DO} /(\mathrm{FW} * \mathrm{RMU} * \mathrm{PERMO} * \mathrm{WCOND}))$

$\mathrm{Q} 1=1.5 \mathrm{D} 0 * \mathrm{~V} /(\mathrm{S} * \mathrm{RMU} * \mathrm{DEL})$

$\mathrm{WL}=\mathrm{CL} / \mathrm{FREQ}$

$W K=2$. D $0 * P I / W L$

$\mathrm{X}=\mathrm{FW} * \mathrm{TR}$

$\mathrm{DEN}=1 . \mathrm{D} 0+\mathrm{X} * \mathrm{X}$

$\mathrm{EPSP}=\mathrm{EPSHP}+($ EPSLP - EPSHP $) / \mathrm{DEN}$

$\mathrm{EPSI}=\mathrm{HCNDSP} /(\mathrm{FW} *$ EPSO $)+($ EPSLP - EPSHP $) * \mathrm{X} / \mathrm{DEN}$

EPSC $=$ CMPLX (EPSP , - EPSI)

REFI $=S Q R T$ (EPSC)

IF (NHSPH . LE. O) THEN $\mathrm{Q} 2 \mathrm{H}=1$. D30

ELSE

CALL ABSORB (HSPRAD, WK, REFI, NT, SIGABS)

$\mathrm{Q} 2 \mathrm{H}=2 . \mathrm{D} 0 * \mathrm{PI} * \mathrm{~V} /(\mathrm{WL} * \mathrm{SIGABS} * \mathrm{NHSPH})$

ENDIF

IF (NOSPH . LE. O) THEN

$\mathrm{Q} 2 \mathrm{O}=1$. D30

ELSE

EPS $I=O C N D S P /(F W * E P S 0)$ 


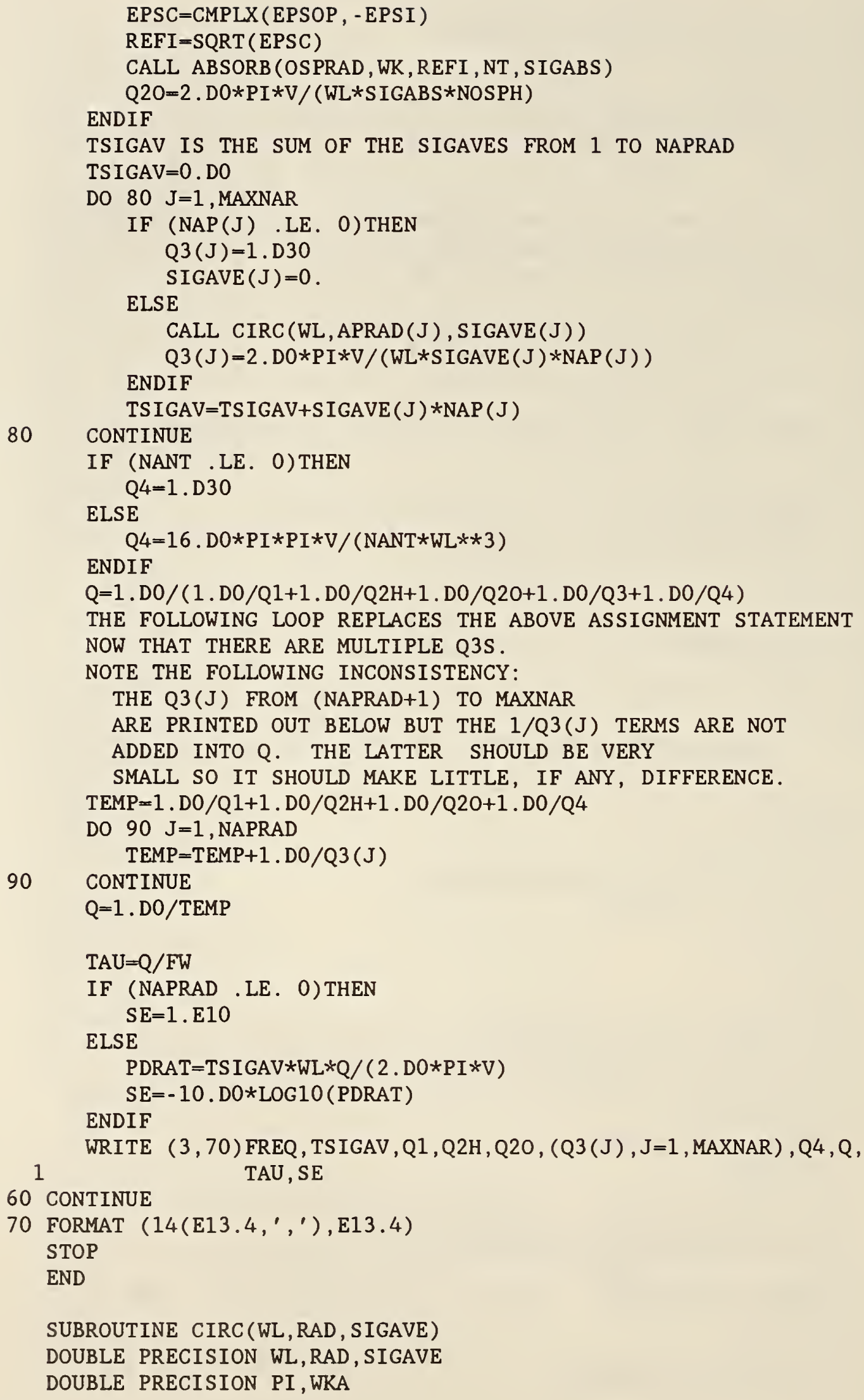


$\mathrm{PI}=3.1415926536$

$\mathrm{PI}=\mathrm{DACOS}(-1 . \mathrm{DO})$

$W K A=2 . D 0 * P I * R A D / W L$

IF (WKA - 1.3DO) $10,20,20$

$10 \mathrm{SIGAVE}=8 . \mathrm{D} 0 *(\mathrm{WKA} * * 4) * \mathrm{RAD} * \mathrm{RAD} /(9 . \mathrm{D} 0 * \mathrm{PI})$

GO TO 30

$20 \mathrm{SIGAVE}=\mathrm{PI} * \mathrm{RAD} * \mathrm{RAD} / 4 . \mathrm{D} 0$

30 RETURN

END

SUBROUTINE ABSORB (A, WK, REFI, NT, SIGABS)

DOUBLE COMPLEX REFI, Y, FJY(50), FH(50), RJY, DRJY, RHX, DRHX, ANUM, ADEN, 1 AN, BN, BNUM , BDEN , FJX ( 50$)$, X, X2 , RJX, DRJX, REFI2 , RAD,

1 RPER, RPAR

DOUBLE PRECISION A, WK, SIGABS

INTEGER NT

DOUBLE PRECISION PI, AVE, SE, SS, TE, TS, QE, QS

INTEGER NTP, NP, N

C

$P I=3.1415926536$

$\mathrm{PI}=\mathrm{DACOS}(-1 . \mathrm{D} 0)$

$\mathrm{X}=\mathrm{WK} * \mathrm{~A}$

C

$\mathrm{NT}=\mathrm{REAL}(\mathrm{X})+3$

$\mathrm{NT}=\mathrm{DBLE}(\mathrm{X})+3$

IF (NT - 49) 20, 20, 30

30 REFI $2=$ REFI $*$ REFI

$\mathrm{RAD}=\mathrm{SQRT}(\mathrm{REFI} 2-.5 \mathrm{D} 0)$

$R P E R=(.707 D 0-R A D) /(.707 D 0+R A D)$

$R P A R=(.707 D 0 * R E F I 2-R A D) /(.707 D 0 * R E F I 2+R A D)$

$\mathrm{AVE}=.5 \mathrm{D} 0 *(\mathrm{ABS}(\mathrm{RPER}) * * 2+\mathrm{ABS}(\mathrm{RPAR}) * * 2)$

$S I G A B S=P I * A * A *(1 . D 0-A V E)$

GO TO 40

$20 \mathrm{Y}=\mathrm{REFI} * \mathrm{X}$

$\mathrm{NTP}=\mathrm{NT}+1$

CALL CSPJ ( $X, N T P, F J X)$

CALL CSPJ (Y, NTP, FJY)

CALL CSPH2 (X, NTP, FH)

$\mathrm{SE}=0$. DO

$\mathrm{SS}=0 . \mathrm{DO}$

DO $10 \mathrm{~N}=1$, NT

$\mathrm{NP}=\mathrm{N}+1$

$\mathrm{RJX}=\mathrm{X} * \mathrm{FJX}(\mathrm{NP})$

$\mathrm{DRJX}=\mathrm{FJX}(\mathrm{NP})+\mathrm{X} * \mathrm{FJX}(\mathrm{N})-\mathrm{NP} * \mathrm{FJX}(\mathrm{NP})$

$R J Y=Y * F J Y(N P)$

$D R J Y=F J Y(N P)+Y * F J Y(N)-N P * F J Y(N P)$

$\mathrm{RHX}=\mathrm{X} * \mathrm{FH}(\mathrm{NP})$

$\mathrm{DRHX}=\mathrm{FH}(\mathrm{NP})+\mathrm{X} * \mathrm{FH}(\mathrm{N})-\mathrm{NP} * \mathrm{FH}(\mathrm{NP})$

ANUM $=$ DRJ $Y * R J X-R E F I * R J Y * D R J X$

$A D E N=D R J Y * R H X-R E F I * R J Y * D R H X$

$\mathrm{AN}=\mathrm{ANUM} / \mathrm{ADEN}$

$B N U M=R E F I * D R J Y * R J X-R J Y * D R J X$

$B D E N=R E F I * D R J Y * R H X-R J Y * D R H X$

$\mathrm{BN}=\mathrm{BNUM} / \mathrm{BDEN}$

C

$\mathrm{TE}=(2 * \mathrm{~N}+1) * \mathrm{REAL}(\mathrm{AN}+\mathrm{BN})$ 


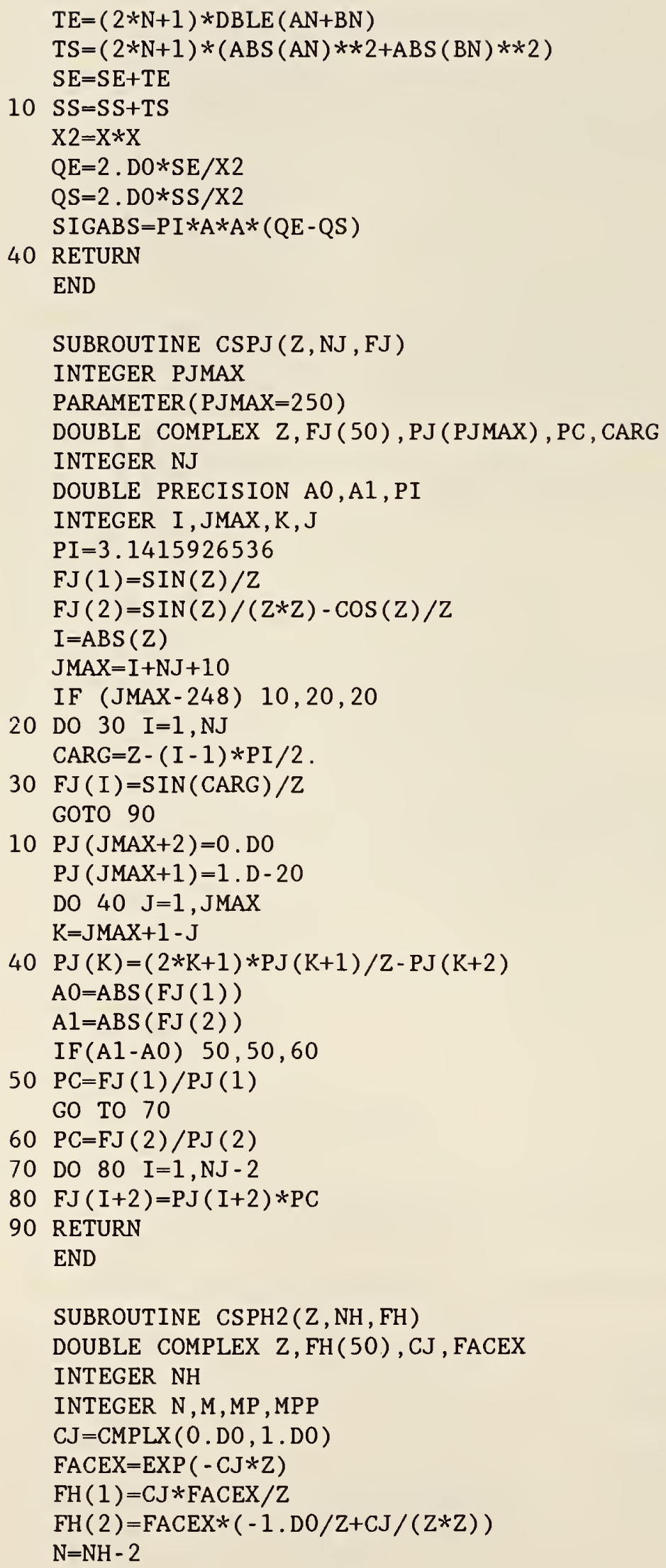


$\operatorname{IF}(\mathrm{N}) \quad 10,10,20$

20 DO $30 \quad M=1, N$

$\mathrm{MP}=\mathrm{M}+1$

$\mathrm{MPP}=\mathrm{M}+2$

$30 \mathrm{FH}(\mathrm{MPP})=(2 * \mathrm{M}+1) * \mathrm{FH}(\mathrm{MP}) / \mathrm{Z}-\mathrm{FH}(\mathrm{M})$

10 CONTINUE

RETURN

END 


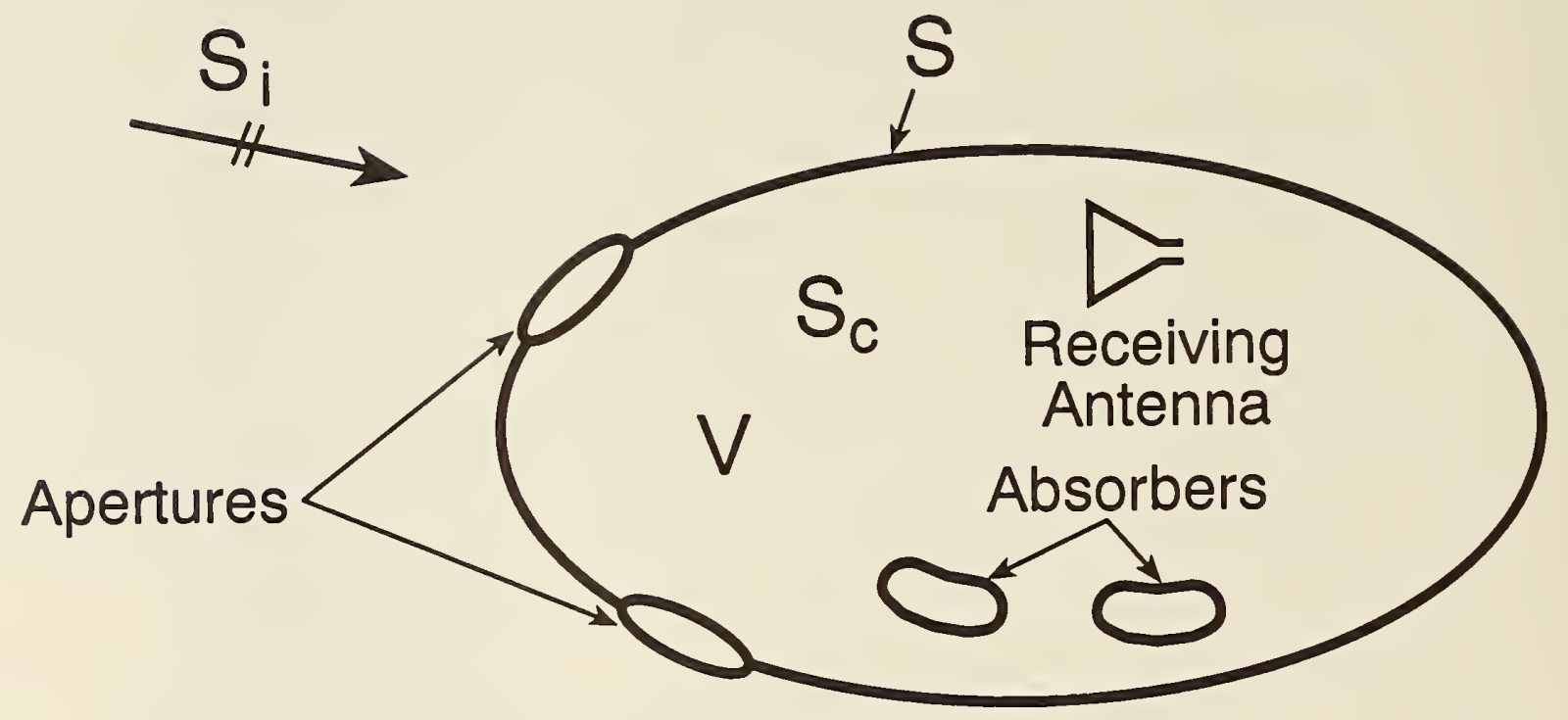

Figure 1. Aperture excitation of a cavity containing absorbers and a receiving antenna. 


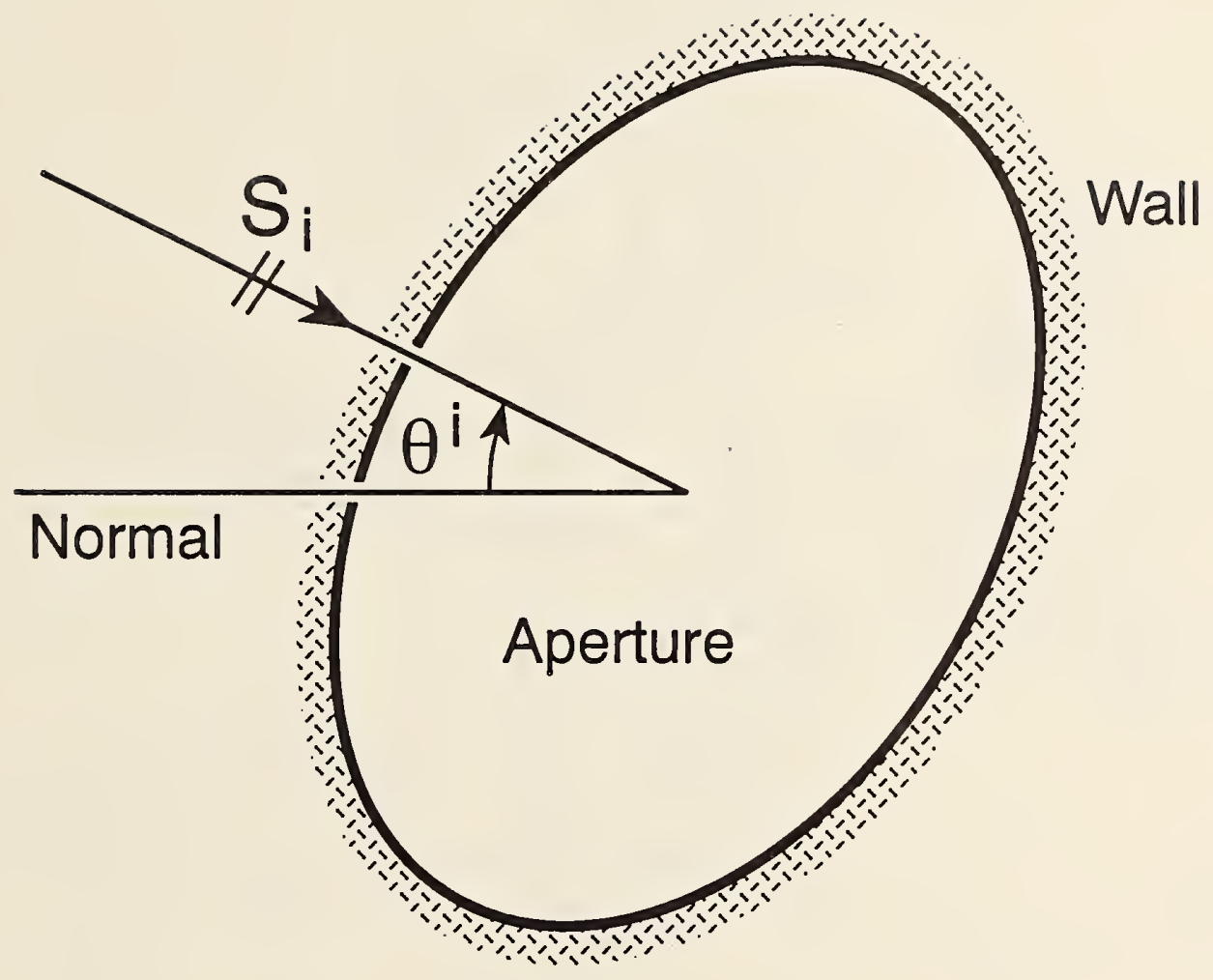

Figure 2. External field incident on an aperture of arbitrary shape. 


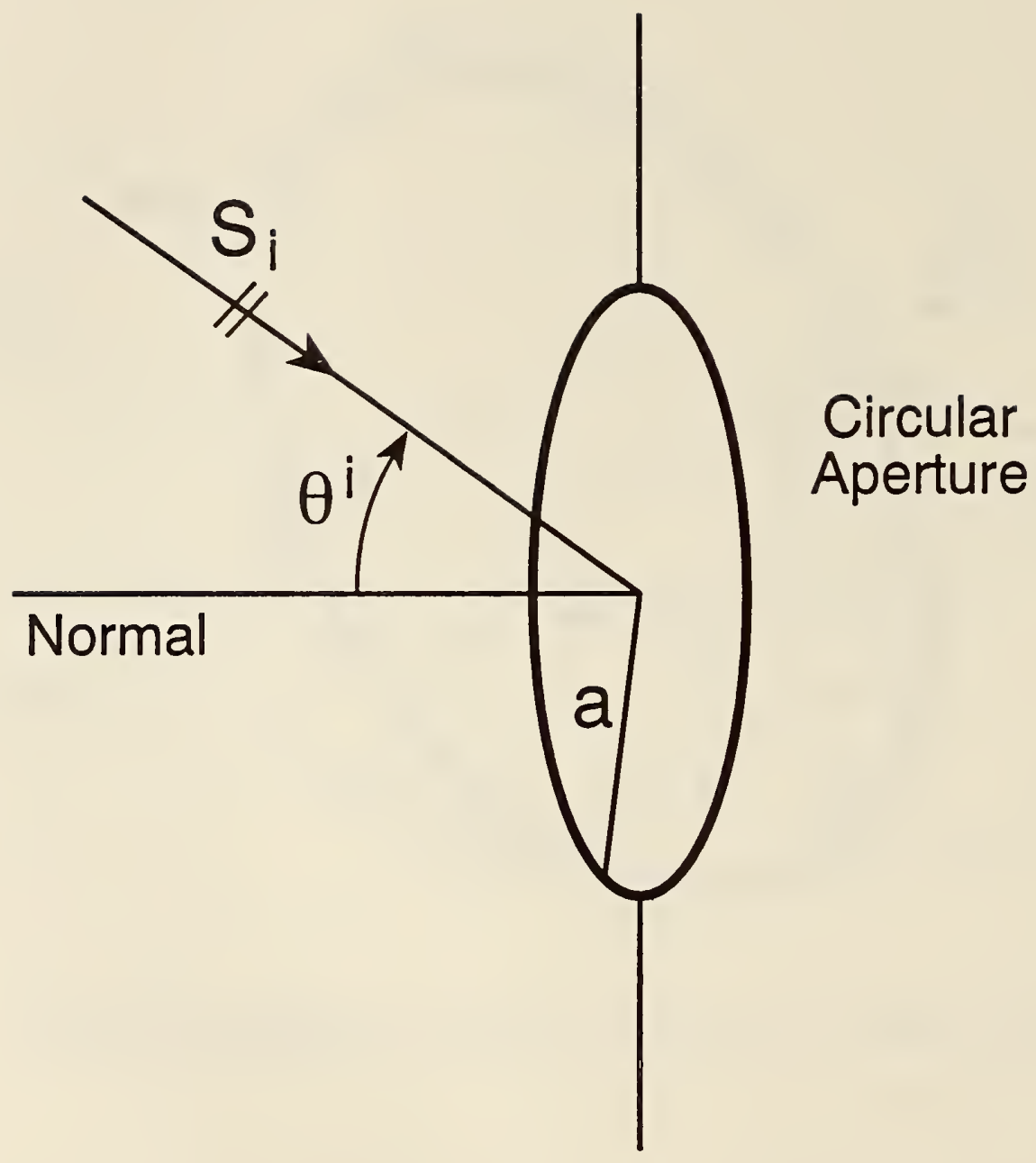

Figure 3. External field incident on a circular aperture of radius a. 


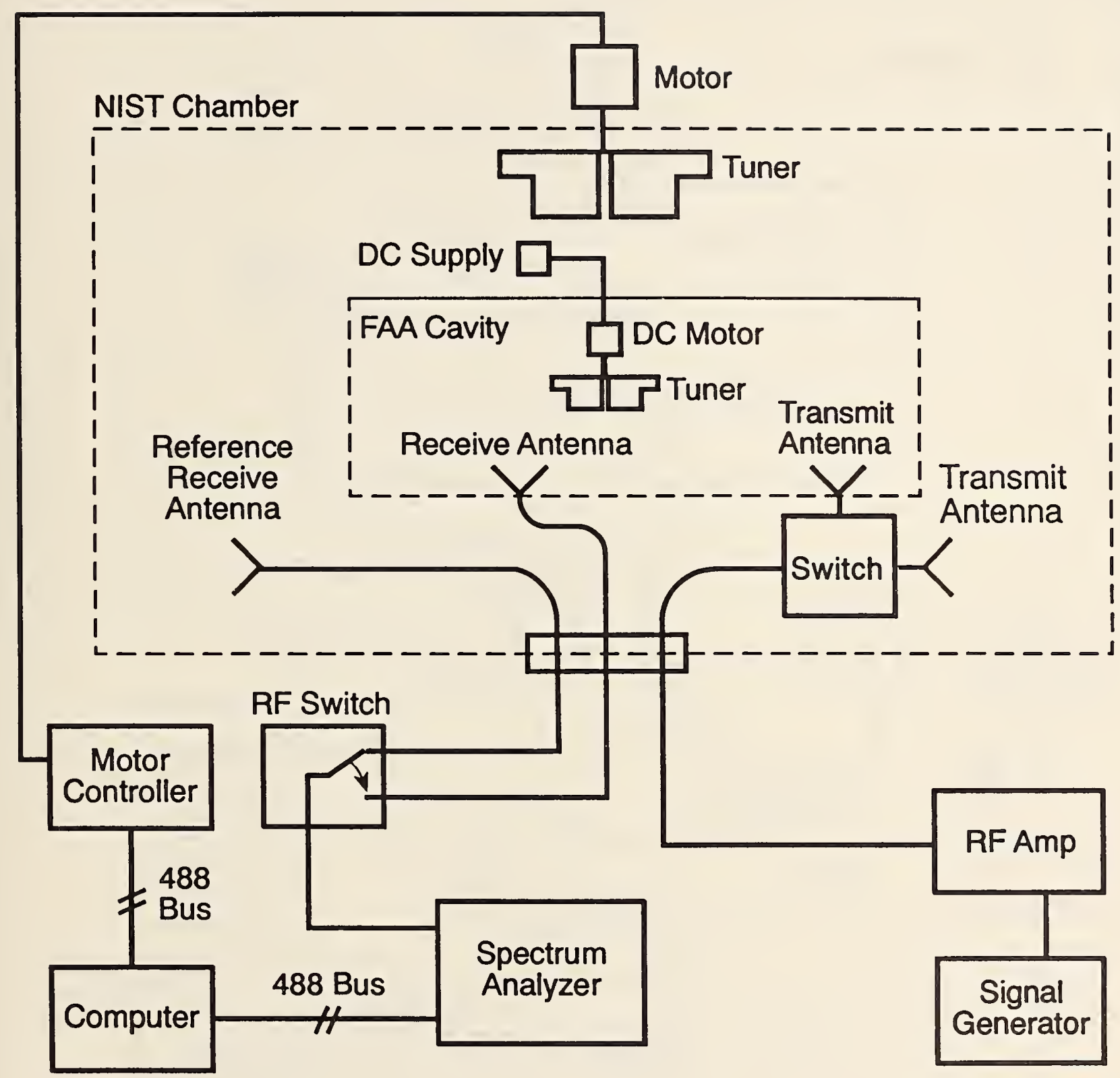

Figure 4. Block diagram of system for measurement of shielding effectiveness. Block diagram of system for measurement of loss is similar; reconnect cable from NIST chamber transmit antenna to FAA cavity transmit antenna. 


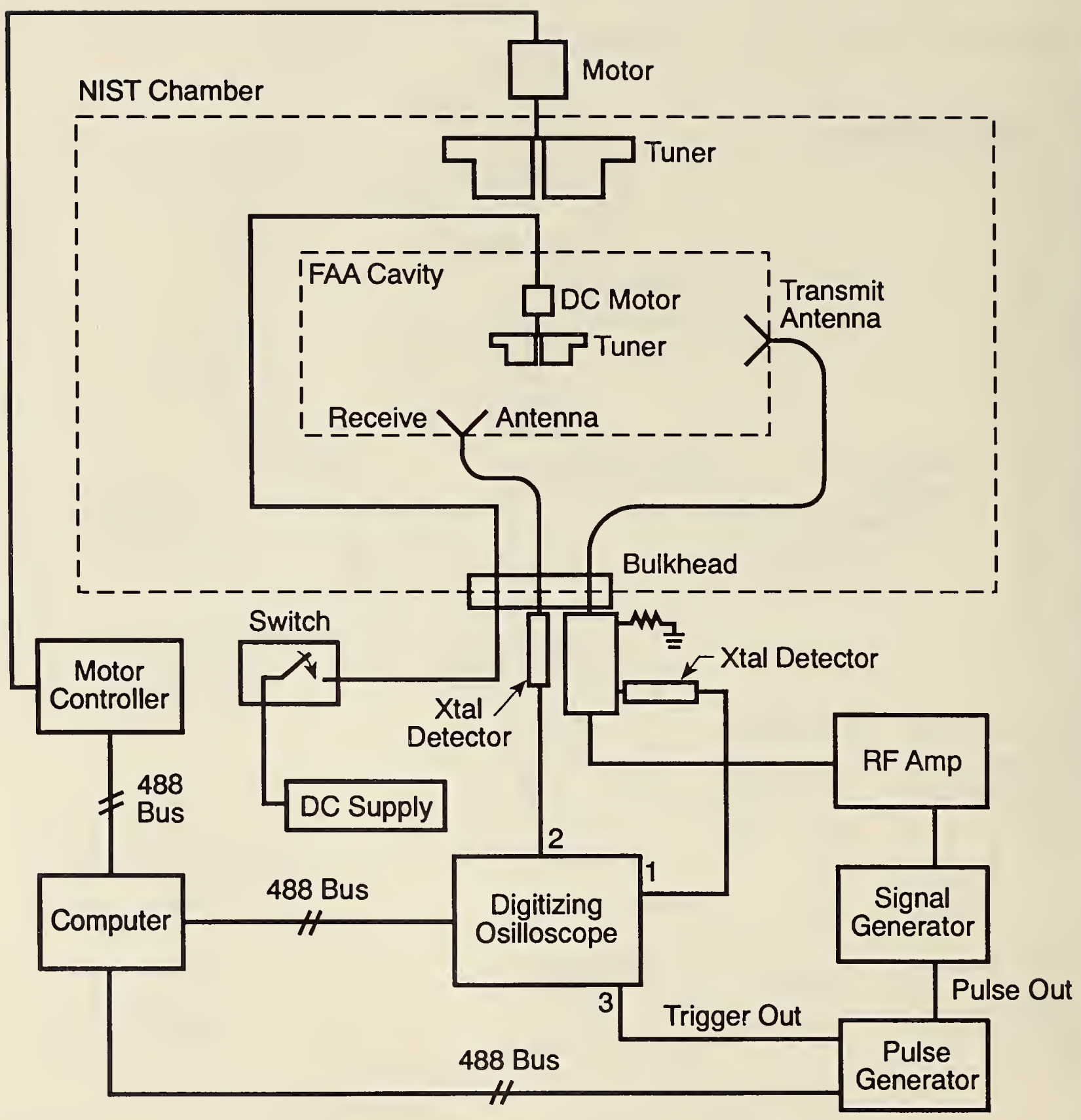

Figure 5. Block diagram of system for measuring time constant. Output data files are used to plot time response curves. One example is shown in figure 6. 


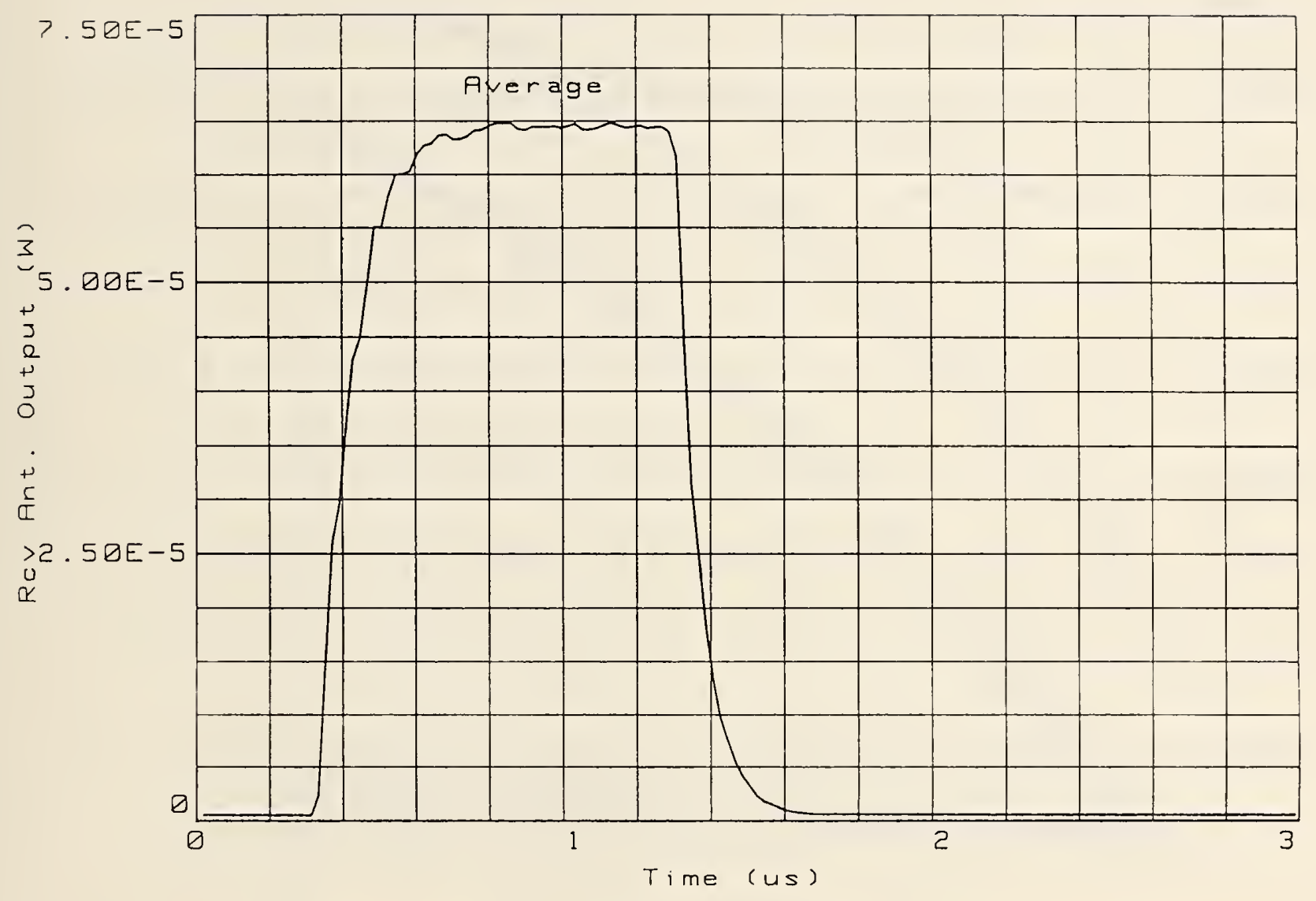

Figure 6. Time response of FAA cavity loaded with one aperture of radius $0.014 \mathrm{~m}$ and one sphere of radius $0.066 \mathrm{~m}$ filled with salt water. This is the average of 200 waveforms from 200 stirrer positions. An exponential, least-squares fit is applied to the decay time to obtain the time constant. Frequency $=18 \mathrm{GHz}$. 


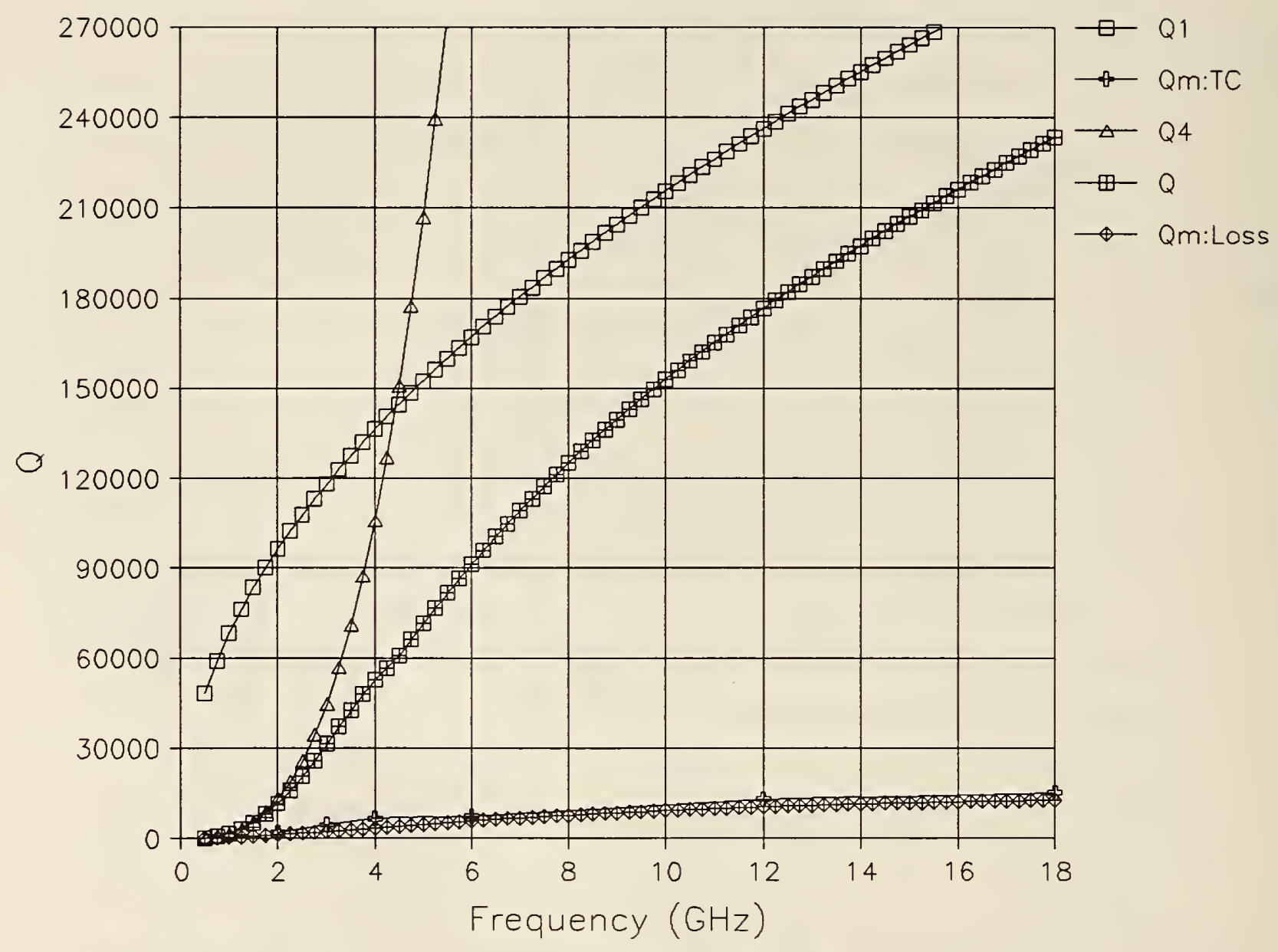

Figure 7. Five plots of $Q$ for the FAA cavity with aperture of radius 0.014 $\mathrm{m}$, two antennas, no absorptive loading, and no corrections. Q1 is the calculated $Q$ from wall losses. Q4 is the calculated $Q$ from two antennas. Q3 is calculated for aperture loading, but the curve is off scale. $Q$ is the composite $Q$ of all calculated Qs from all loss mechanisms in the model. Qm-TC is Q calculated from measured time constant values. Qm-Loss is Q calculated from measured loss values. There is a large discrepancy between measured and calculated values. See text and later figures for explanation. 


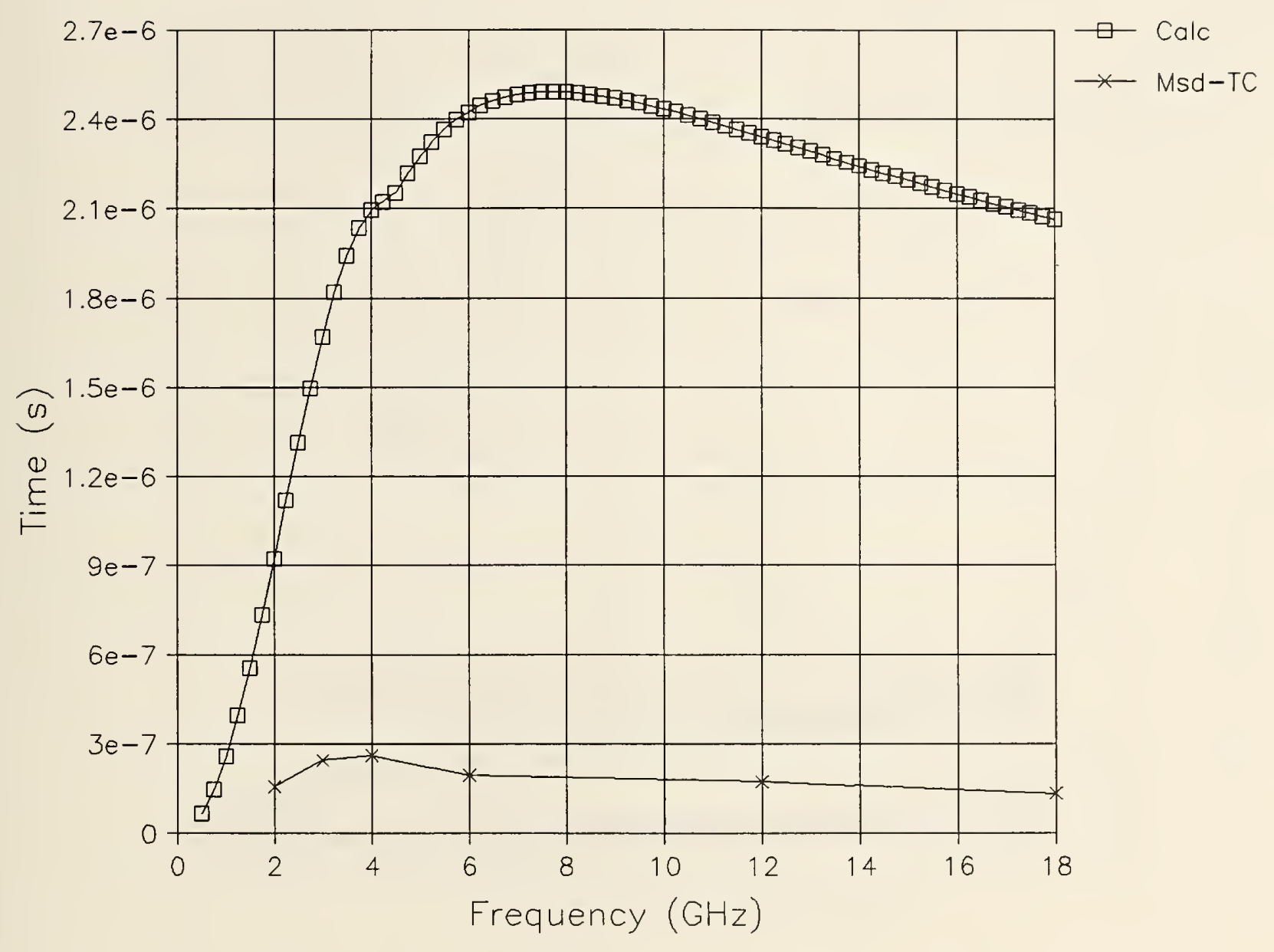

Figure 8. Calculated and measured time constant values for the FAA cavity with aperture of radius $0.014 \mathrm{~m}$, two antennas, no absorptive loading, and no corrections. Squares indicate calculated and $\times s$ indicate measured values. There is a large discrepancy between the two. See text and later figures for explanation. 


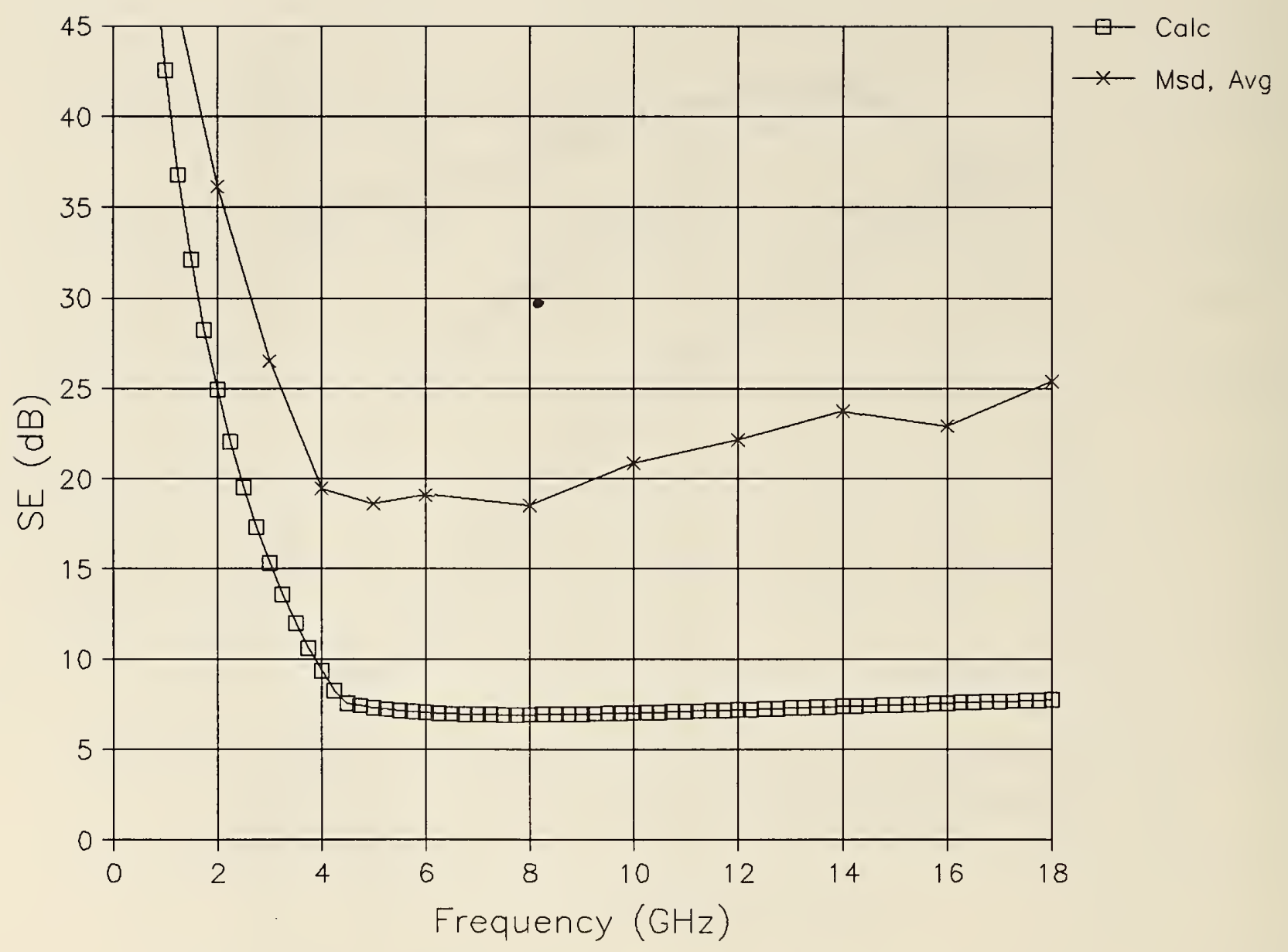

Figure 9. Calculated and measured values of SE for the FAA cavity with aperture of radius $0.014 \mathrm{~m}$, two antennas, no absorptive loading, and no corrections. There is a large discrepancy between the two. See text and later figures for explanation. 


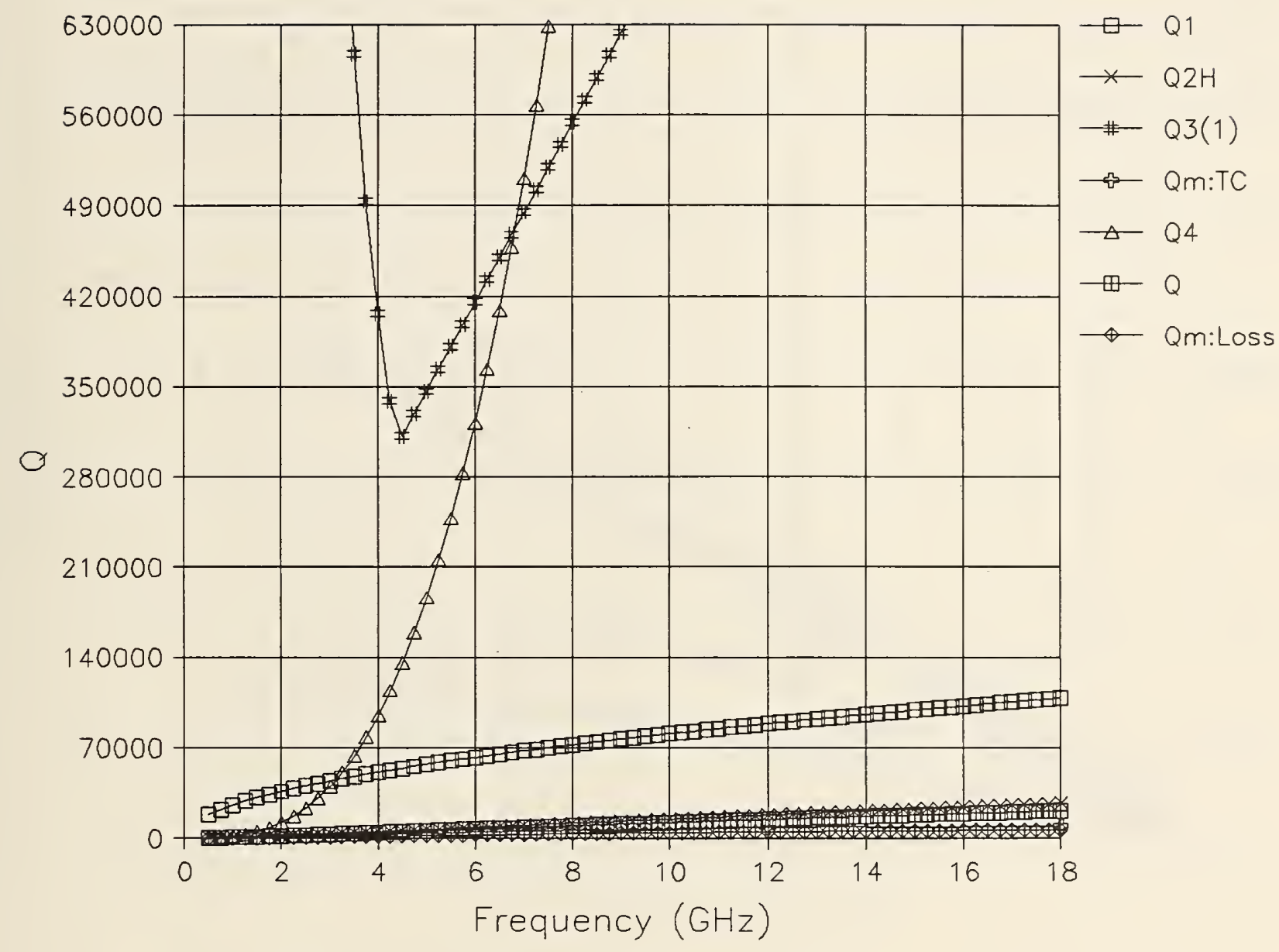

Figure 10. Seven plots of $Q$ for the FAA cavity with aperture of radius 0.014 $\mathrm{m}$, two antennas, one sphere of radius $0.066 \mathrm{~m}$ filled with salt water for absorptive loading. Conductivity (decreased by 4), volume ( $10 \%$ decrease), and surface area (20\% increase) corrections are applied. Q1 is the calculated Q from wall losses, Q4 is the calculated Q from two antennas, Q3 is the aperture loading. Q2H, Q, Qm-TC, and Qm-loss are compressed at the bottom of the figure. See figure 11 for expanded scale. 


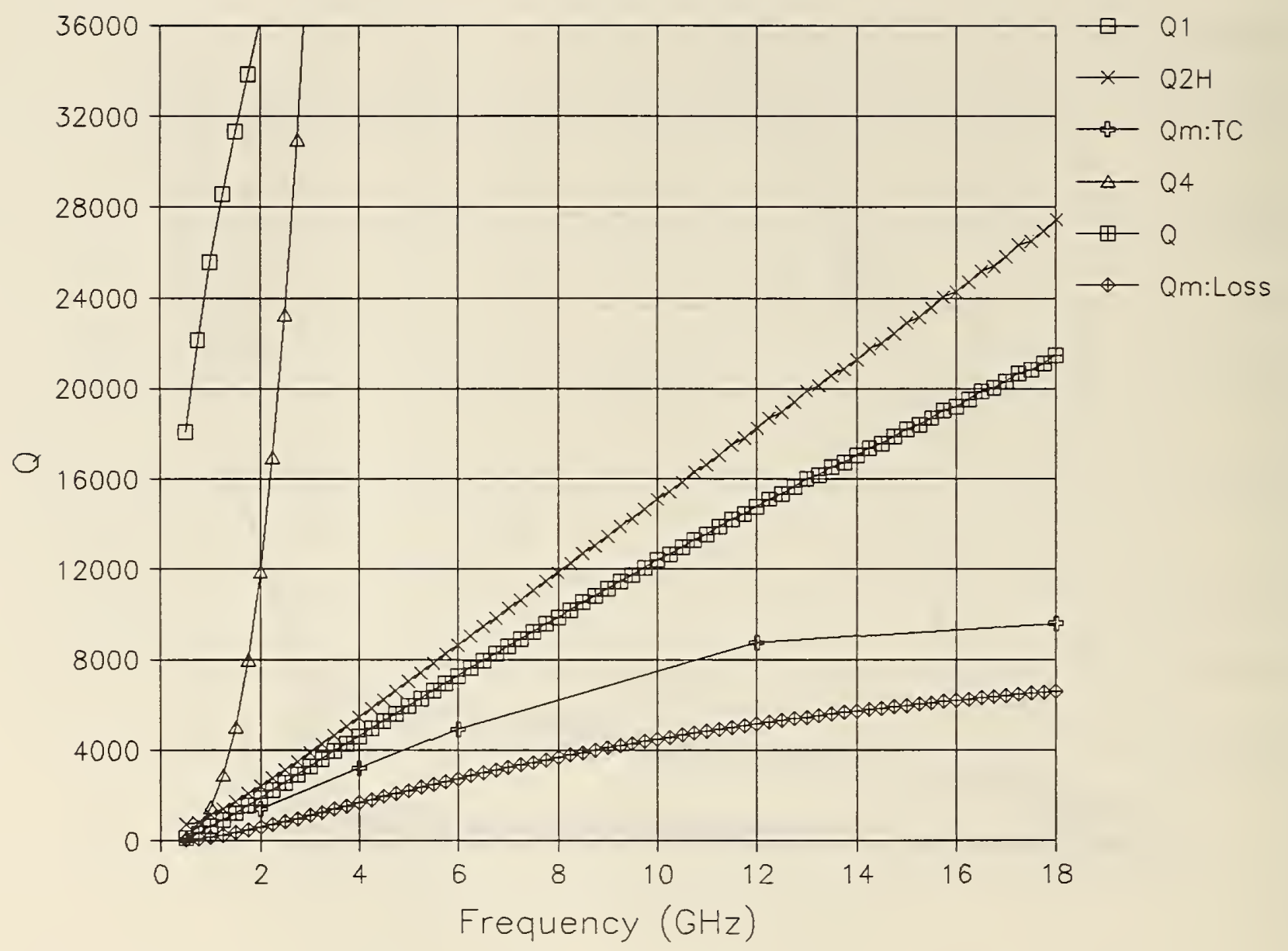

Figure 11. Six plots of $Q$ for the FAA cavity with aperture of radius 0.014 $\mathrm{m}$, two antennas, one sphere of radius $0.066 \mathrm{~m}$ filled with salt water for absorptive loading. Conductivity (decreased by 4), volume (10\% decrease), and surface area (20\% increase) corrections are applied. Q1 is the calculated Q from wall losses. Q4 is the calculated Q from two antennas. Q3 is the aperture loading, off scale. $\mathrm{Q} 2 \mathrm{H}$ is the calculated $\mathrm{Q}$ for absorptive loading of the sphere of salt water, $Q$ is the composite $Q$ of all calculated Qs from all loss mechanisms in the model, Qm-TC is Q calculated from measured time constant values. Qm-Loss is Q calculated from measured loss values. 


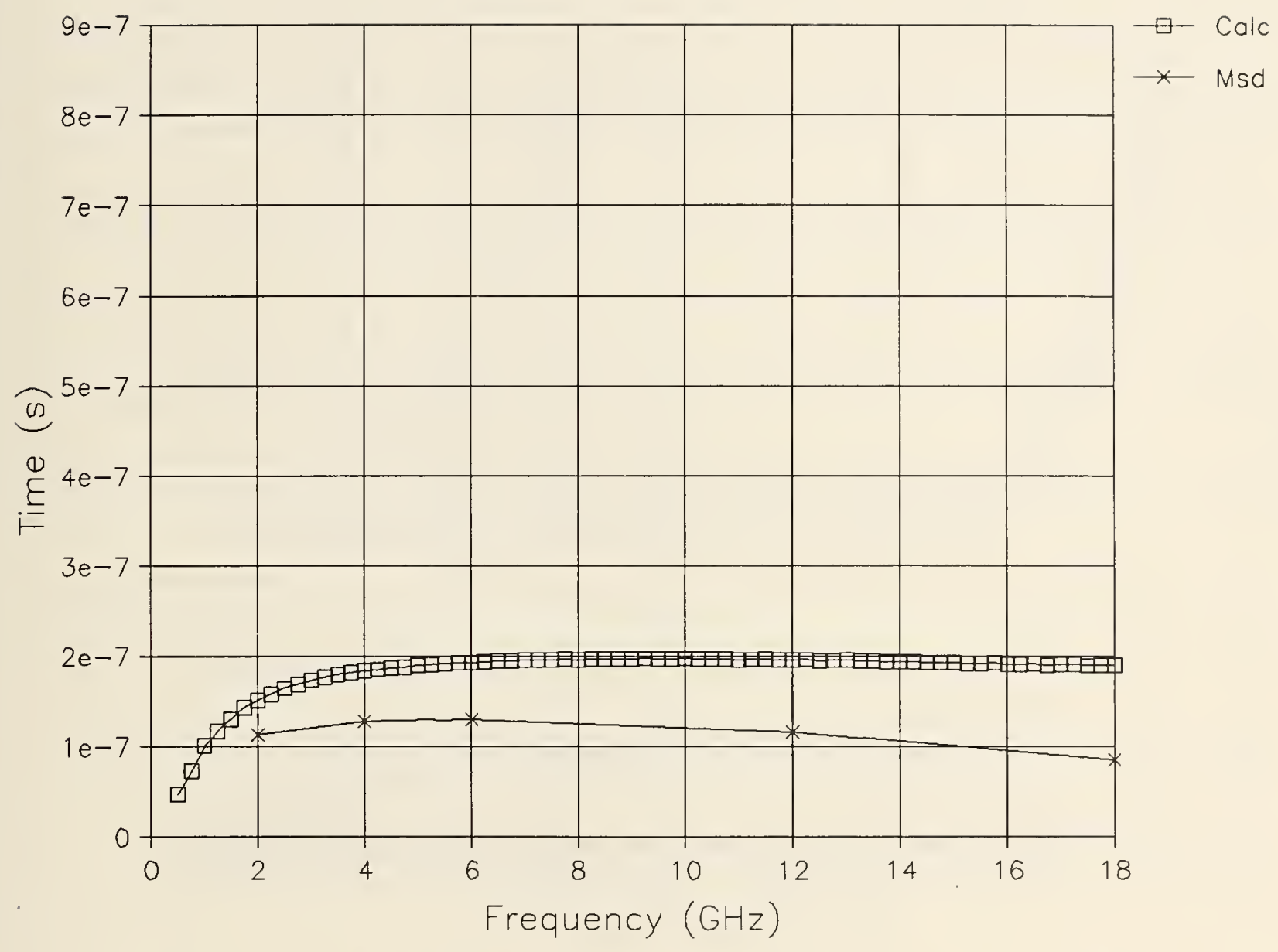

Figure 12. Calculated and measured time constant values for the FAA cavity with aperture of radius $0.014 \mathrm{~m}$, two antennas, one sphere of radius $0.066 \mathrm{~m}$ filled with salt water for absorptive loading. Conductivity (decreased by 4), volume (10\% decrease), and surface area (20\% increase) corrections are applied. Squares indicate calculated and $x$ s indicate measured values. There is much better agreement between the measured and calculated values. 


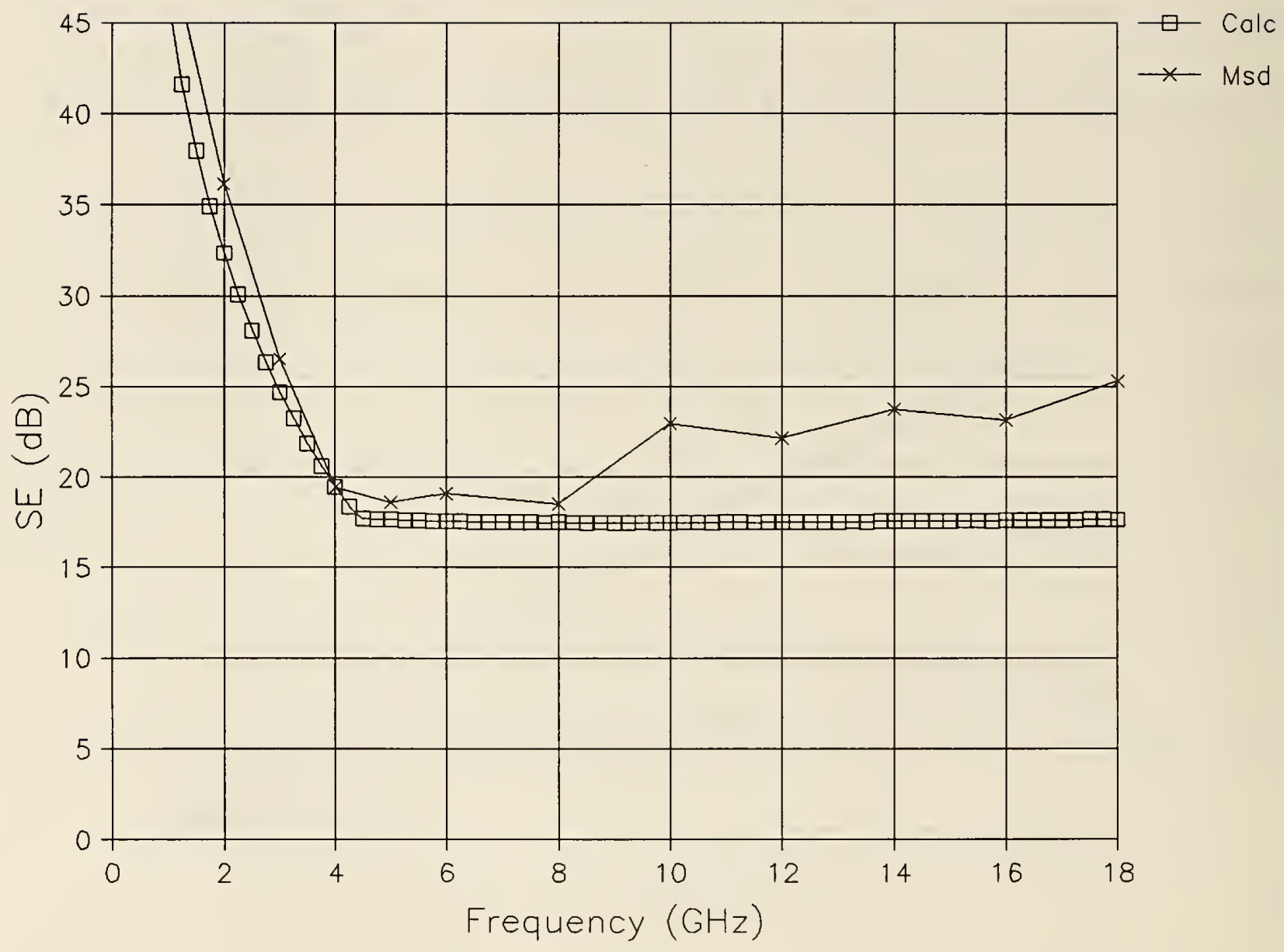

Figure 13. Calculated and measured values of SE for the FAA cavity with aperture of radius $0.014 \mathrm{~m}$, two antennas, one sphere of radius $0.066 \mathrm{~m}$ filled with salt water for absorptive loading. Conductivity (decreased by 4 ), volume (10\% decrease), and surface area (208 increase) corrections are applied. There is much better agreement between the measured and calculated values. 


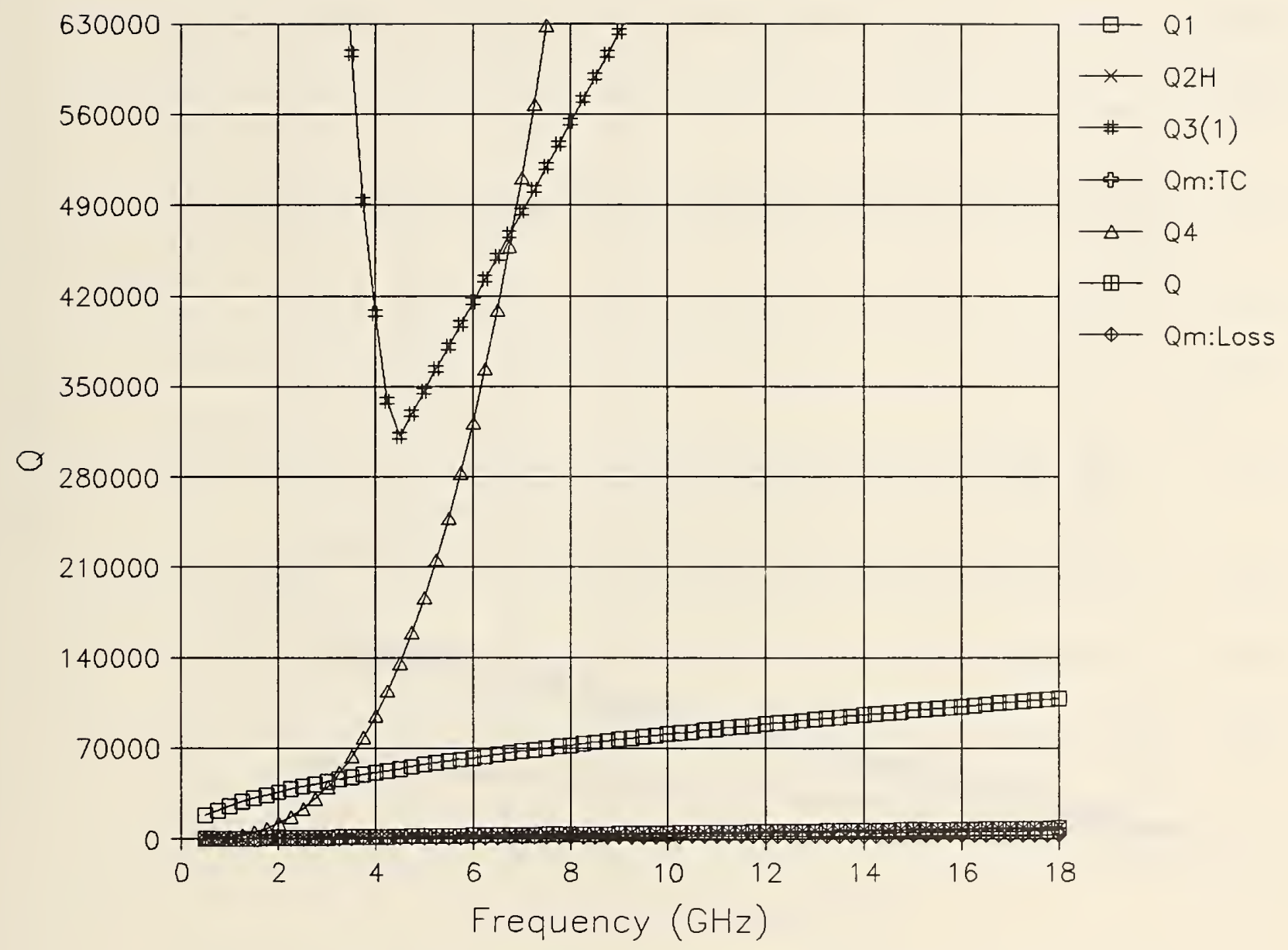

Figure 14. Seven plots of $Q$ for the FAA cavity with aperture of radius 0.014 $\mathrm{m}$, two antennas, three spheres of radius $0.066 \mathrm{~m}$ filled with salt water for absorptive loading. Conductivity (decreased by 4), volume (10\% decrease), and surface area (20\% increase) corrections are applied. Q1 is the calculated Q from wall losses. Q4 is the calculated Q from two antennas. Q3 is the calculated $Q$ from aperture loading. Q2H, Q, Qm-TC, and Qm-loss are compressed at the bottom of the figure. See expanded scale in figure 15. 


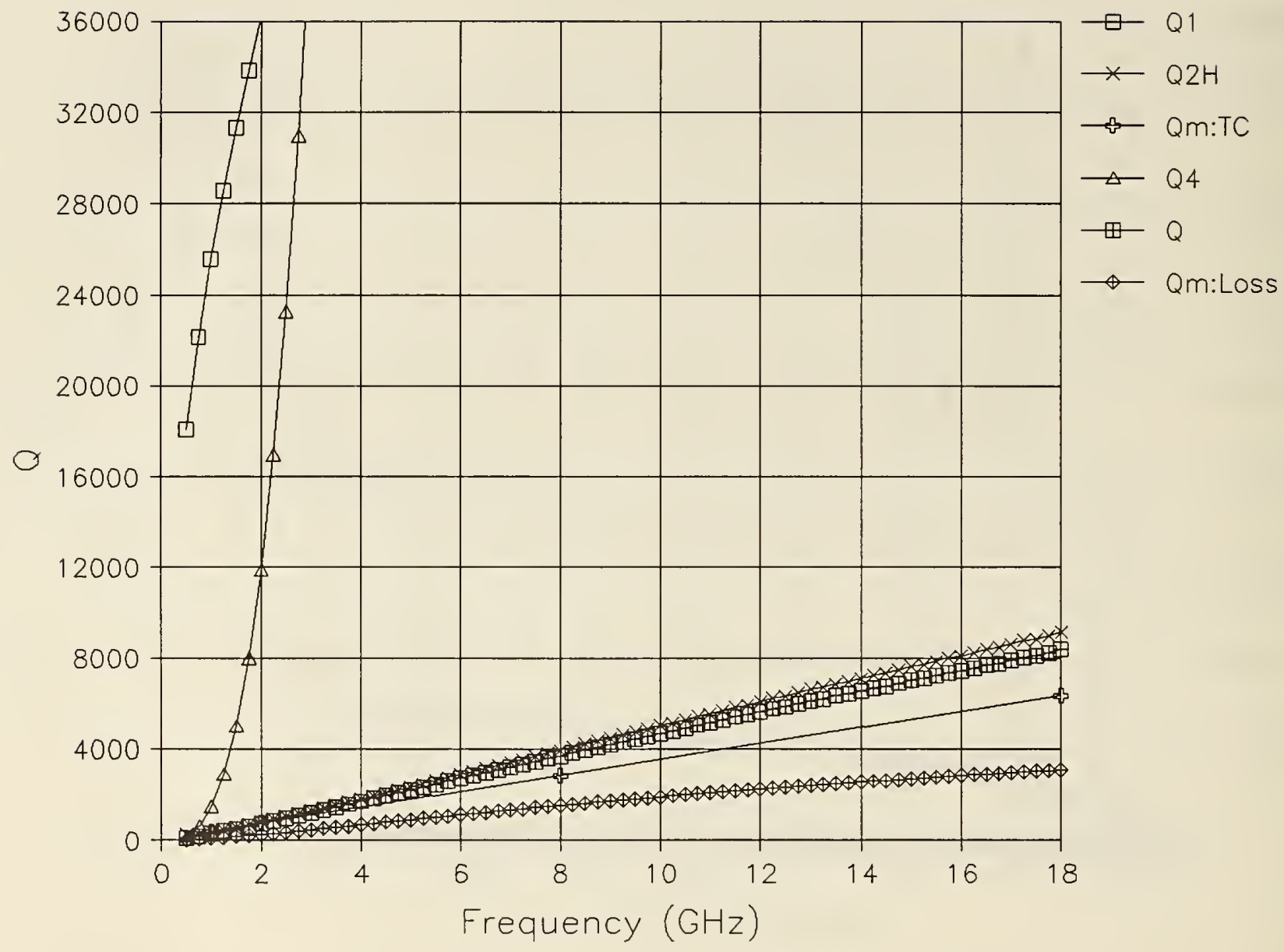

Figure 15. Six plots of $Q$ for the FAA cavity with aperture of radius 0.014 $\mathrm{m}$, two antennas, three spheres of radius $0.066 \mathrm{~m}$ filled with salt water for absorptive loading. Conductivity (decreased by 4), volume (108 decrease), and surface area (208 increase) corrections are applied. Q1 is the calculated $Q$ from wall losses. Q4 is the calculated $Q$ from two antennas. Q3 is the calculated $Q$ from aperture loading, off scale. Q2H is the calculated $Q$ for absorptive loading of the spheres of salt water, $Q$ is the composite $Q$ of all calculated Qs from all loss mechanisms in the model, Qm-TC is Q calculated from measured time constant values. Qm-Loss is Q calculated from measured loss values. There is good agreement between measured and calculated values. 


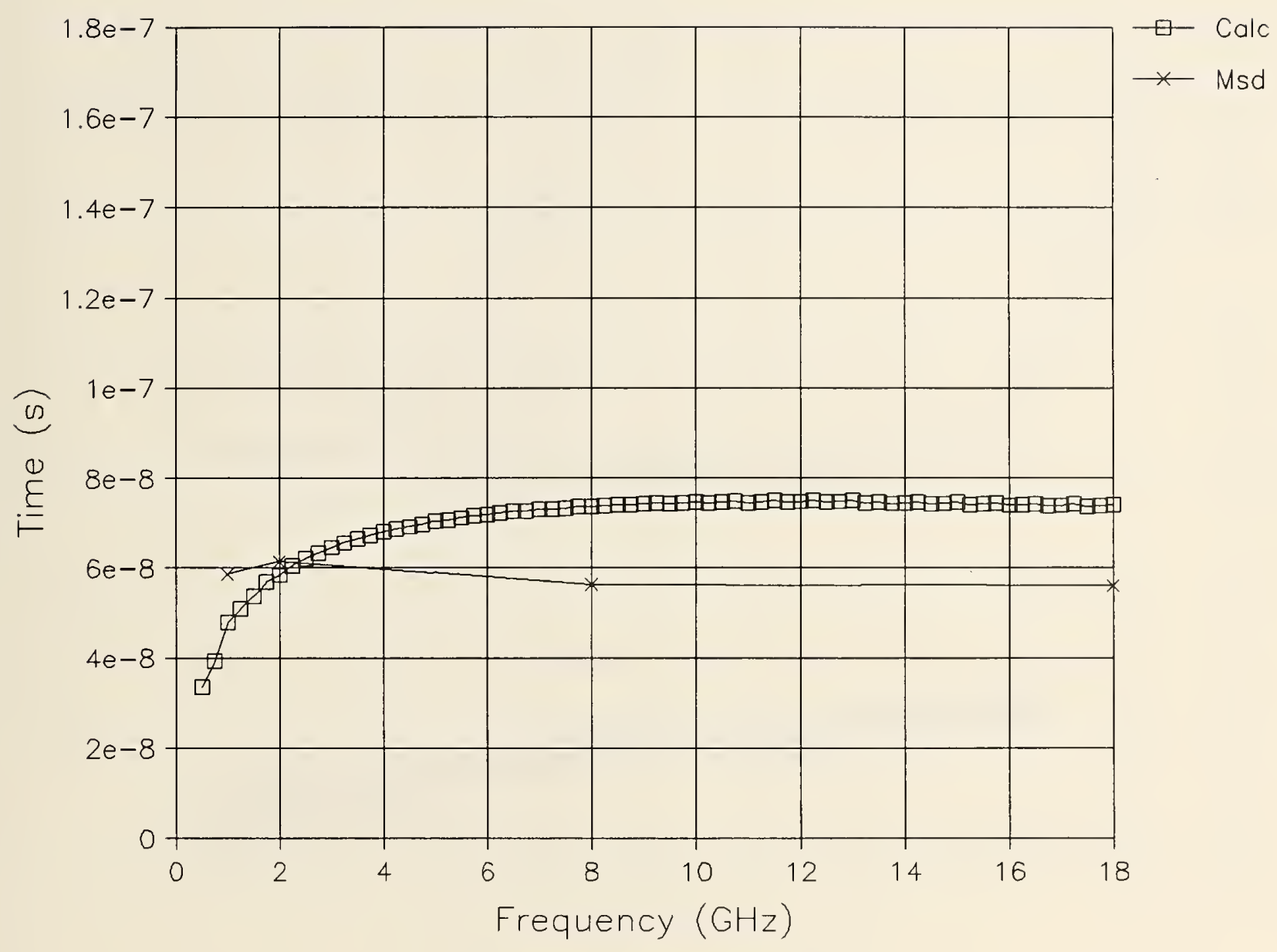

Figure 16. Calculated and measured time constant values for the FAA cavity with aperture of radius $0.014 \mathrm{~m}$, two antennas, three spheres of radius $0.066 \mathrm{~m}$ filled with salt water for absorptive loading. Conductivity (decreased by 4 ), volume ( $10 \%$ decrease), and surface area (208 increase) corrections are applied. Squares indicate calculated and $x$ s indicate measured values. There is good agreement between measured and calculated values. 


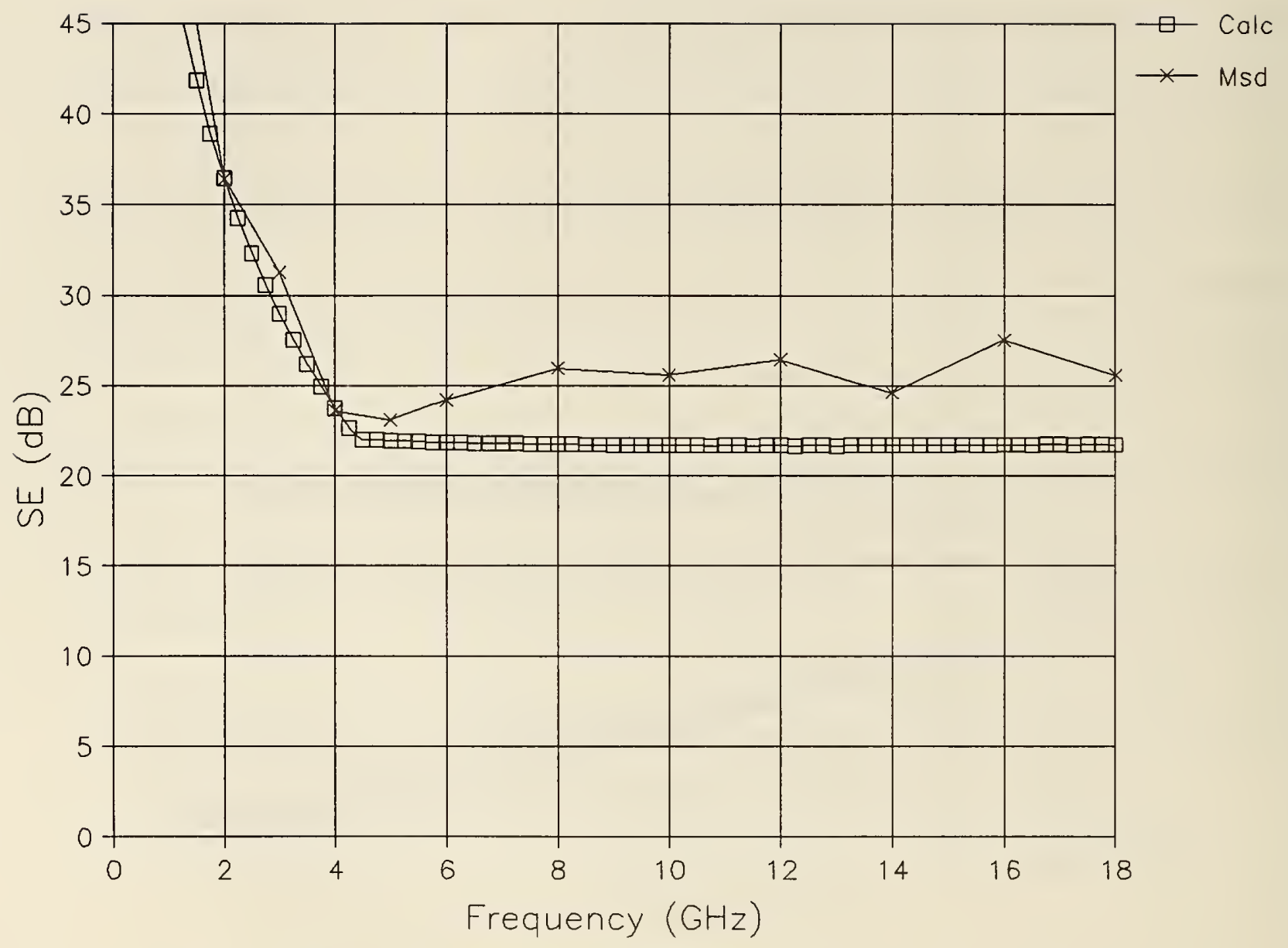

Figure 17. Calculated and measured values of SE for the FAA cavity with aperture of radius $0.014 \mathrm{~m}$, two antennas, three spheres of radius $0.066 \mathrm{~m}$ filled with salt water for absorptive loading. Conductivity (decreased by 4 ), volume (108 decrease), and surface area (208 increase) corrections are applied. There is good agreement between the measured and calculated values. 


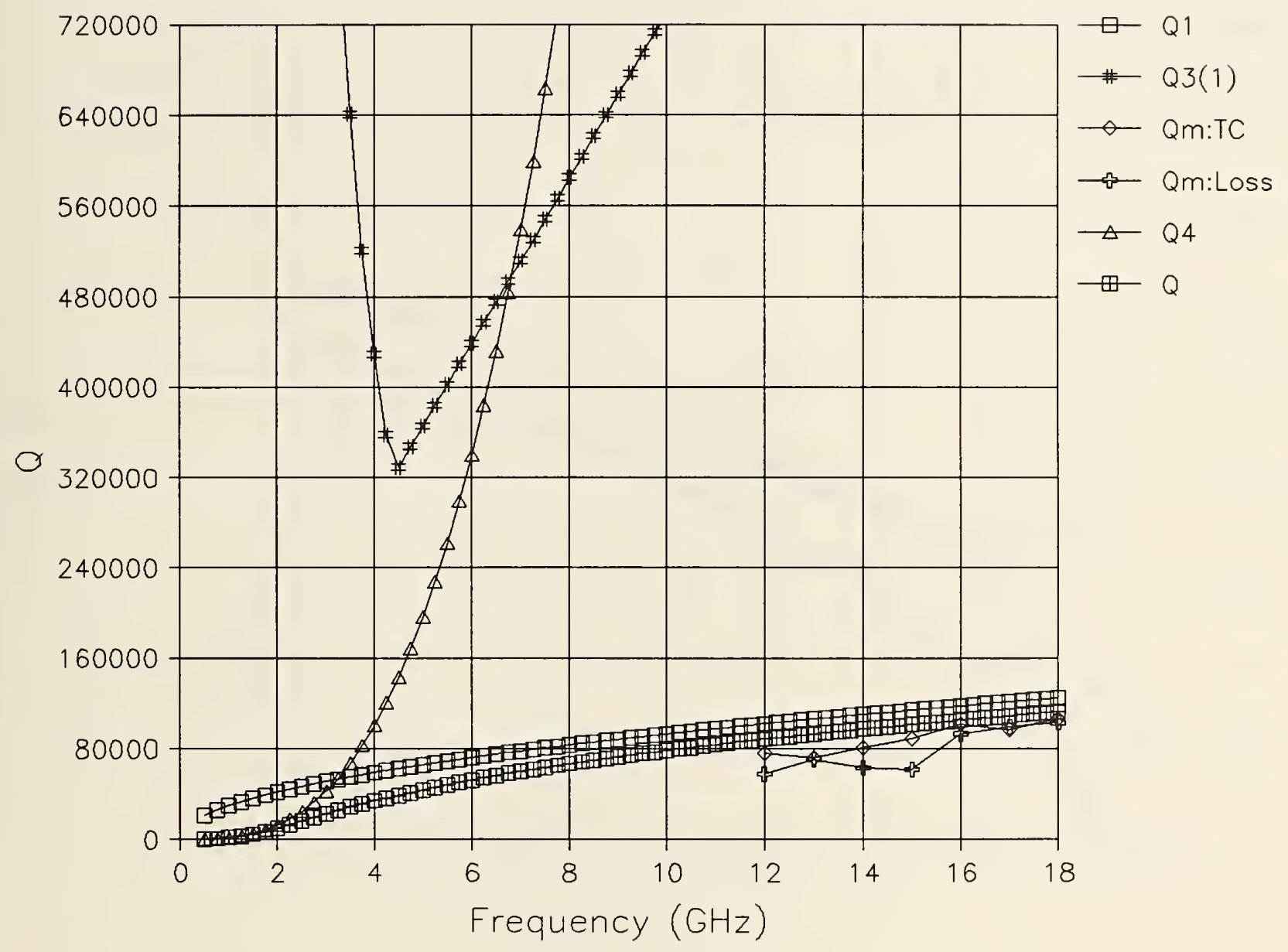

Figure 18. Six plots of $Q$ for the FAA cavity with aperture of radius 0.014 $\mathrm{m}$, two antennas, no absorption. Conductivity (decreased by 4), volume ( $5 \%$ decrease), and surface area (10\% increase) corrections are applied. Standard gain horns were used for measurements from 12 to $18 \mathrm{GHz}$. Q1 is the calculated Q from wall losses. Q4 is the calculated $Q$ from two antennas. Q3 is the calculated $Q$ from aperture loading. Q, Qm-TC, and Qm-loss are compressed at the bottom of the figure. See figure 19 for expanded scale. 


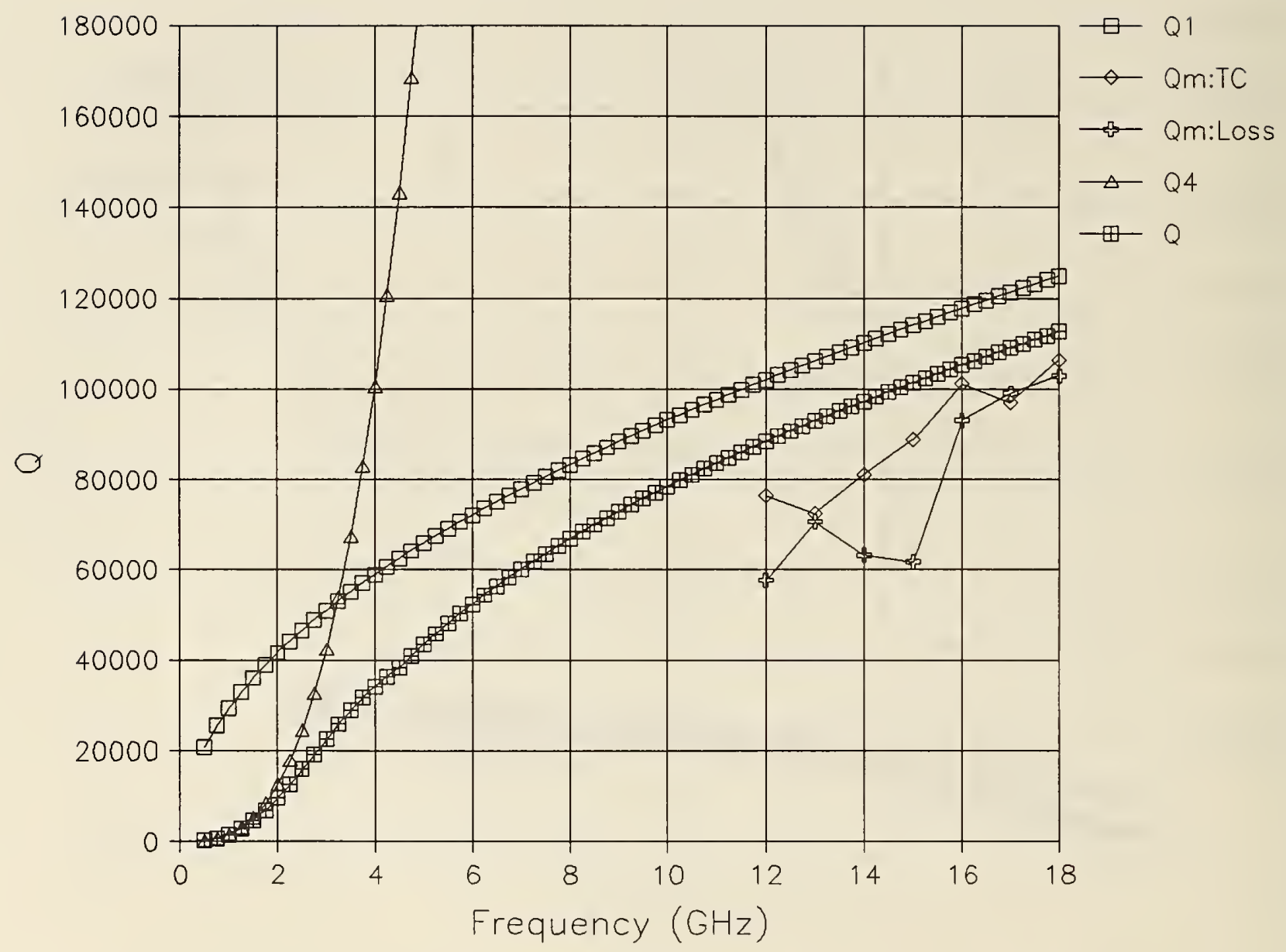

Figure 19. Five plots of $Q$ for the FAA cavity with aperture of radius 0.014 $m$, two antennas, no absorptive loading. Conductivity (decreased by 4), volume ( $5 \%$ decrease), and surface area ( $10 \%$ increase) corrections are applied. Standard gain horns were used for measurements from 12 to $18 \mathrm{GHz}$. Q1 is the calculated Q from wall losses. Q4 is the calculated $Q$ from two antennas. Q3 is the calculated $Q$ from aperture loading, off scale, $Q$ is the composite $Q$ of all calculated $Q$ s from all loss mechanisms in the model, Qm-TC is Q calculated from measured time constant values, Qm-Loss is $Q$ calculated from measured loss values. There is very good agreement between measured and calculated values; the differences are within 208 , well within measurement system uncertainties. 


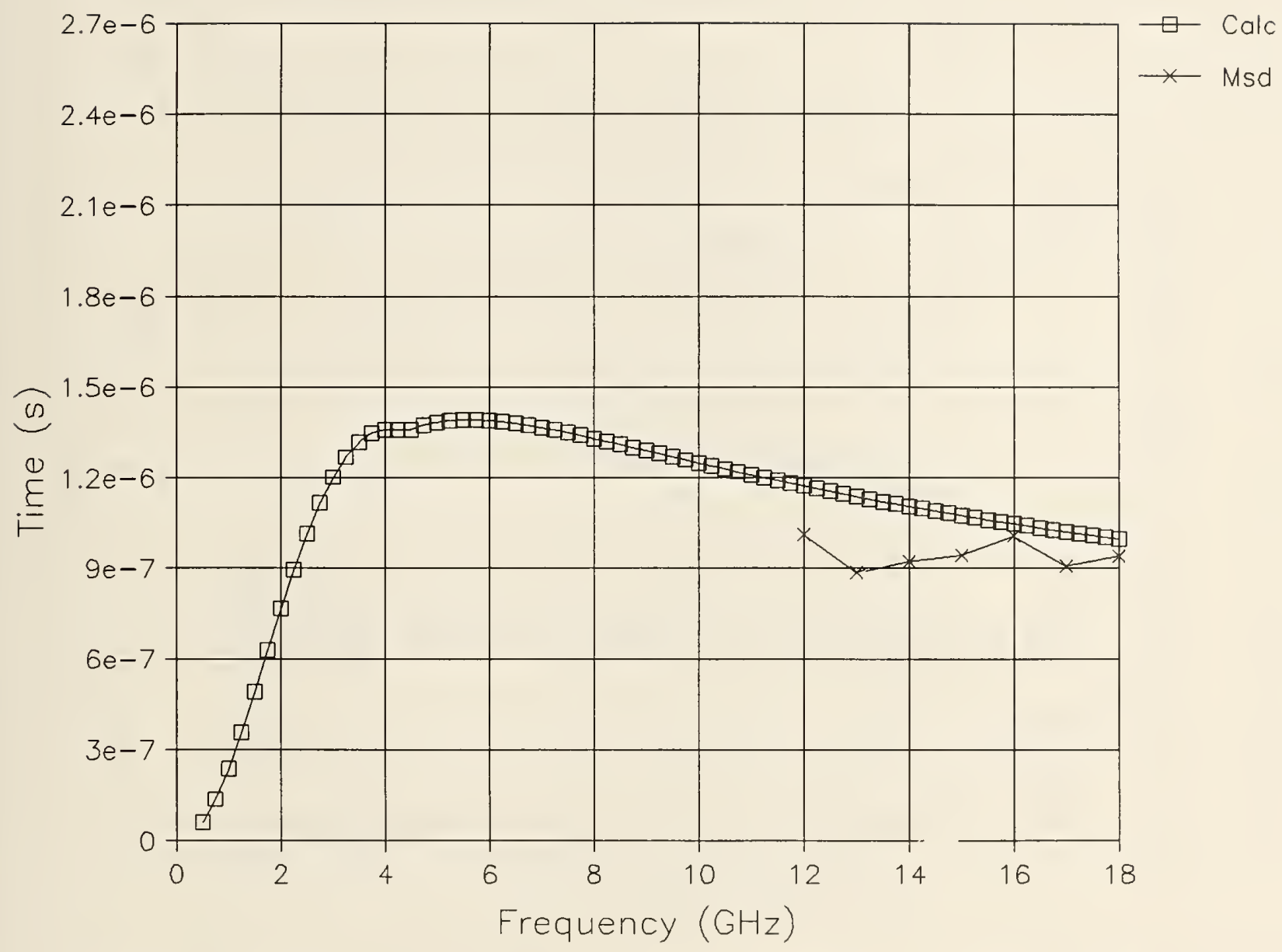

Figure 20. Calculated and measured time constant values for the FAA cavity with aperture of radius $0.014 \mathrm{~m}$, two antennas, no absorptive loading. Conductivity (decreased by 4), volume (58 decrease), and surface area ( $10 \%$ increase) corrections are applied. Squares indicate calculated and $x s$ indicate measured values. There is very good agreement between measured and calculated values; the differences are within measurement system uncertainties. 


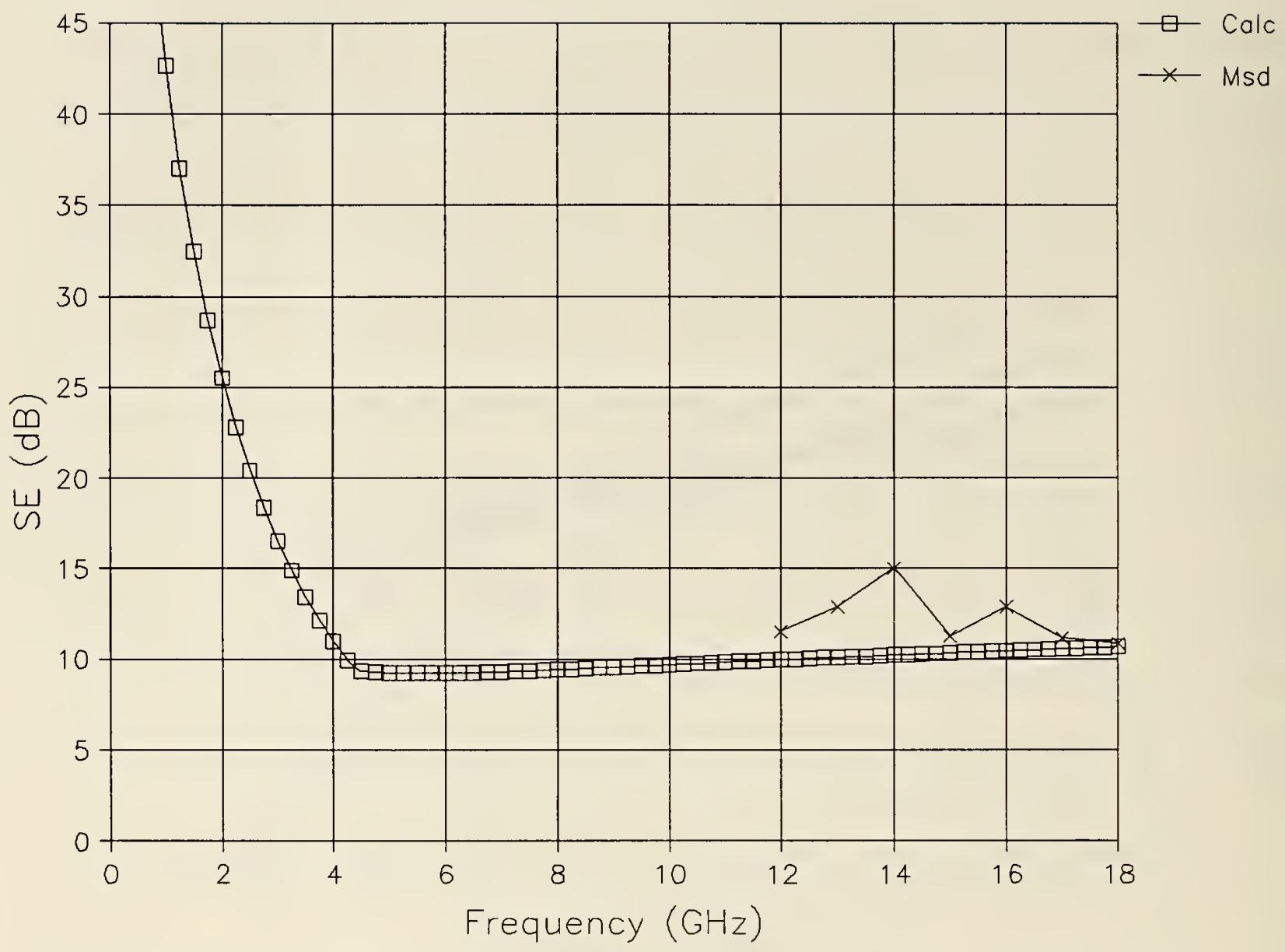

Figure 21. Calculated and measured values of SE for the FAA cavity with aperture of radius $0.014 \mathrm{~m}$, two antennas, no absorptive loading. Conductivity (decreased by 4), volume ( 58 decrease), and surface area (108 increase) corrections are applied. There is good agreement between the measured and calculated values; differences are within the measurement system uncertainties. 


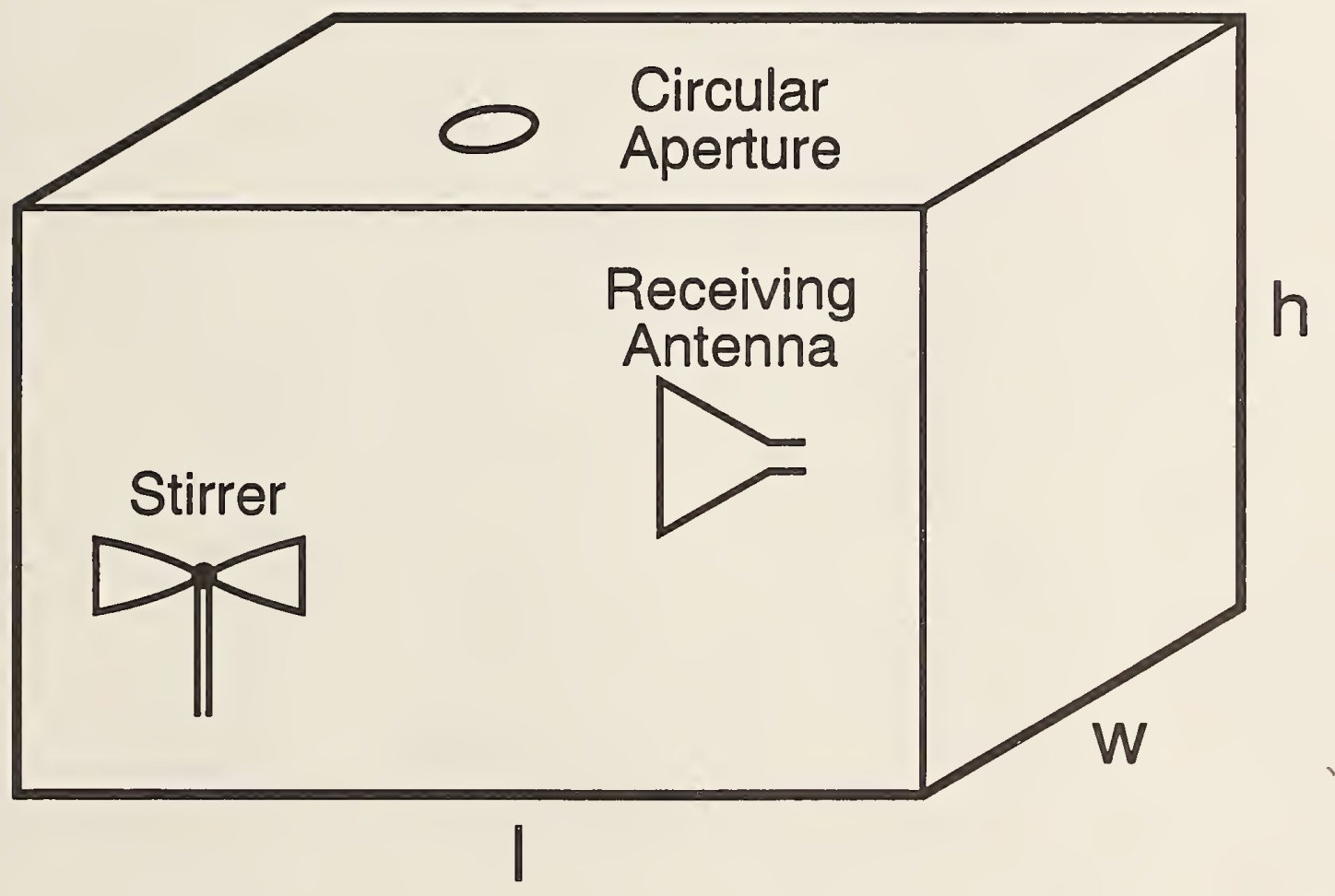

Figure 22. NSWC rectangular cavity with a circular aperture. A mode stirrer and a receiving antenna are located inside. 


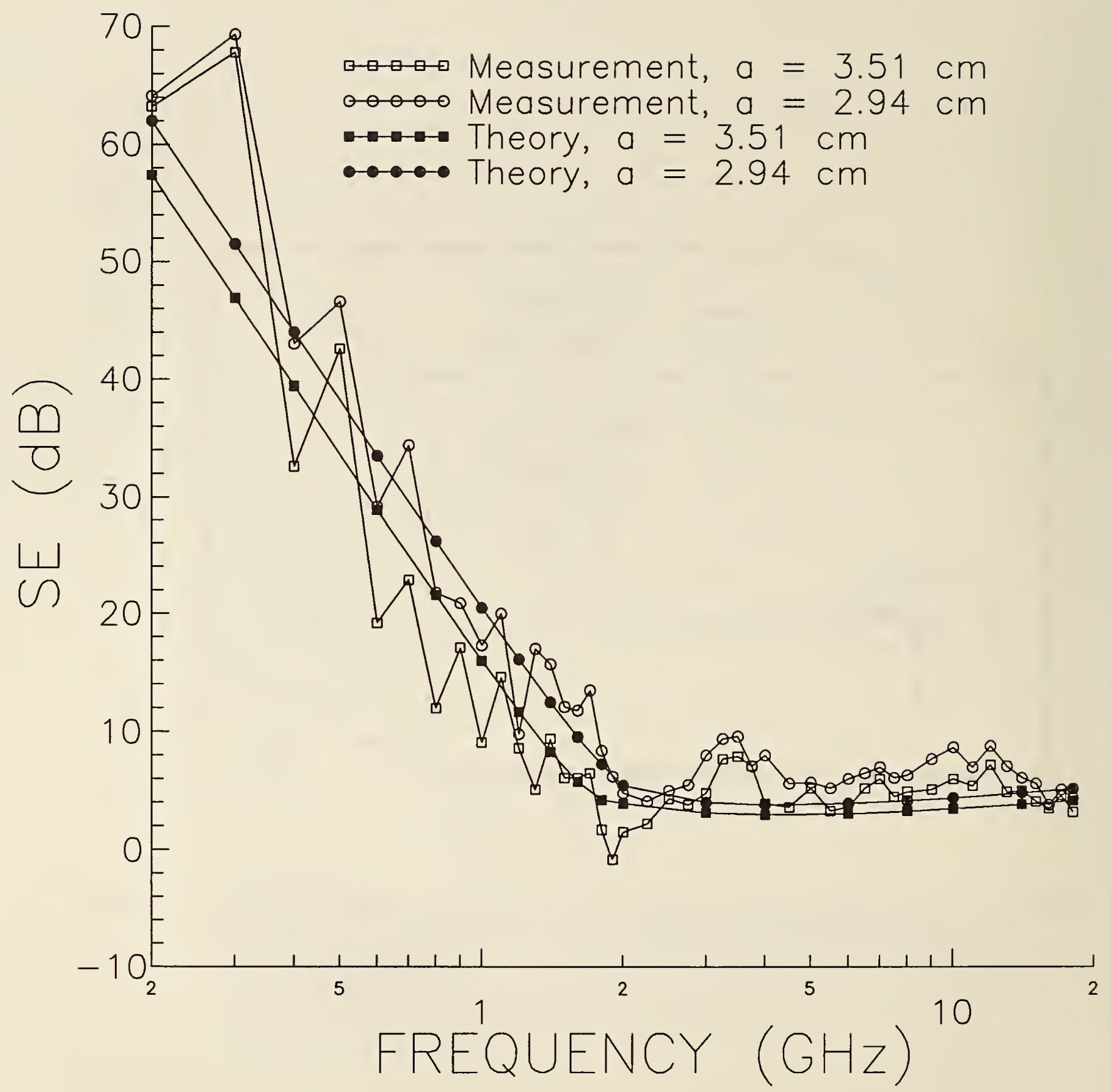

Figure 23. Comparison of calculated and measured SE for the NSWC rectangular cavity with a circular aperture. 


\section{NIST Technical Publications}

\section{Periodical}

Journal of Research of the National Institute of Standards and Technology-Reports NIST research and development in those disciplines of the physical and engineering sciences in which the Institute is active. These include physics, chemistry, engineering, mathematics, and computer sciences. Papers cover a broad range of subjects, with major emphasis on measurement methodology and the basic technology underlying standardization. Also included from time to time are survey articles on topics closely related to the Institute's technical and scientific programs. Issued six times a year.

\section{Nonperiodicals}

Monographs-Major contributions to the technical literature on various subjects related to the Institute's scientific and technical activities.

Handbooks-Recommended codes of engineering and industrial practice (including safety codes) developed in cooperation with interested industries, professional organizations, and regulatory bodies.

Special Publications-Include proceedings of conferences sponsored by NIST, NIST annual reports, and other special publications appropriate to this grouping such as wall charts, pocket cards, and bibliographies.

Applied Mathematics Series-Mathematical tables, manuals, and studies of special interest to physicists, engineers, chemists, biologists, mathematicians, computer programmers, and others engaged in scientific and technical work.

National Standard Reference Data Series-Provides quantitative data on the physical and chemical properties of materials, compiled from the world's literature and critically evaluated. Developed under a worldwide program coordinated by NIST under the authority of the National Standard Data Act (Public Law 90-396). NOTE: The Journal of Physical and Chemical Reference Data (JPCRD) is published bimonthly for NIST by the American Chemical Society (ACS) and the American Institute of Physics (AIP). Subscriptions, reprints, and supplements are available from ACS, 1155 Sixteenth St., NW, Washington, DC 20056 .

Building Science Series-Disseminates technical information developed at the Institute on building materials, components, systems, and whole structures. The series presents research results, test methods, and performance criteria related to the structural and environmental functions and the durability and safety characteristics of building elements and systems.

Technical Notes-Studies or reports which are complete in themselves but restrictive in their treatment of a subject. Analogous to monographs but not so comprehensive in scope or definitive in treatment of the subject area. Often serve as a vehicle for final reports of work performed at NIST under the sponsorship of other government agencies.

Voluntary Product Standards-Developed under procedures published by the Department of Commerce in Part 10, Title 15, of the Code of Federal Regulations. The standards establish nationally recognized requirements for products, and provide all concerned interests with a basis for common understanding of the characteristics of the products. NIST administers this program in support of the efforts of private sector standardizing organizations.

Consumer Information Series-Practical information, based on NIST research and experience, covering areas of interest to the consumer. Easily understandable language and illustrations provide useful background knowledge for shopping in today's technological marketplace.

Order the above NIST publications from: Superintendent of Documents, Government Printing Office, Washington, DC 20402.

Order the following NIST publications-FIPS and NISTIRs-from the National Technical Information Service, Springfield, VA 22161.

Federal Information Processing Standards Publications (FIPS PUB)-Publications in this series collectively constitute the Federal Information Processing Standards Register. The Register serves as the official source of information in the Federal Government regarding standards issued by NIST pursuant to the Federal Property and Administrative Services Act of 1949 as amended, Public Law 89-306 (79 Stat. 1127), and as implemented by Executive Order 11717 (38 FR 12315, dated May 11, 1973) and Part 6 of Title 15 CFR (Code of Federal Regulations).

NIST Interagency Reports (NISTIR)-A special series of interim or final reports on work performed by NIST for outside sponsors (both government and non-government). In general, initial distribution is handled by the sponsor; public distribution is by the National Technical Information Service, Springfield, VA 22161 , in paper copy or microfiche form. 


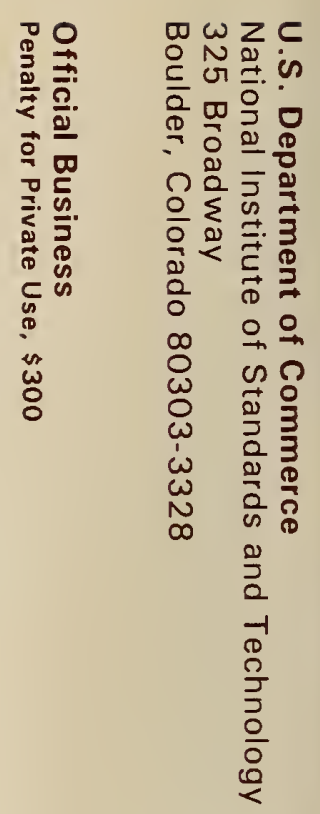

